\title{
3. Gewaltberichte im Fernsehen: journalistische Deutungs- und Handlungsmuster
}

In den vorigen Kapiteln ist erörtert worden, dass Fernsehjournalistinnen und Fernsehjournalisten ihre Themenauswahl und die Machart ihrer Beiträge vor allem daraus ableiten, wie sie die Bedürfnisse ihres Publikums deuten. Dabei speist sich ihr Publikumsbild aus Mutmaßungen über das Publikum, die sie auf die Daten der Sehbeteiligung und auf die Einschätzungen und das Handeln anderer Medienschaffender stützen, kaum aber auf einen direkten Kontakt mit ihrer Zielgruppe. Der Stand der Forschung wird nunmehr auf das Medienthema Gewaltkriminalität bezogen, um die Forschungsfrage zu beantworten: Von welchen Publikumsbedürfnissen hinsichtlich der Berichterstattung über Gewaltkriminalität gehen Fernsehschaffende aus?

\subsection{Die vermutete Neigung des Publikums zu Gewaltberichterstattung}

Bislang sind die Motive der Verantwortlichen von Kriminalitätsberichterstattung kaum erforscht. Eine Reihe von Untersuchungen schließt aus inhaltsanalytischen Befunden auf journalistische Absichten und impliziert, dass das Medienthema Gewalt als Schlüssel zum Publikumserfolg gilt. So werten Groebel und Gleich die Qualität und Quantität aller Aggressions- und Bedrohungshandlungen in jeweils einer künstlichen Programmwoche von ARD, ZDF, RTL, SAT.1, Tele5 und ProSieben aus und kommen zu der Annahme, Gewalt im Fernsehen sei ein „Anziehungsfaktor“ und werde von den Programmverantwortlichen unabhängig von der Relevanz der Beitragsthemen eingesetzt (Groebel und Gleich 1993: 102). Auch Bruns und Marcinkowski unterstellen den Verantwortlichen von Nachrichtensendungen, sie nutzten Gewalt gezielt, um „Involvement“ zu erzeugen, also die Zuschauer emotional zu bewegen und an den Sender $\mathrm{zu}$ binden (Bruns und Marcinskowsi 1997: 222f., vgl. Fröhlich 1998: 376).

Pfeiffer, Windzio und Kleimann kritisieren vor allem eine Dramatisierung von Gewaltkriminalität aufgrund von ,quotenorientierter Berichterstattung auch über Kriminalität" (Pfeiffer, Windzio und Kleimann 2005, vgl. Windzio und Kleimann 2006: 196, Windzio, Simonson, Pfeiffer und Kleimann 2007: 6, Norek 2005: 20, Groebel und Gleich 1993: 20, Kepplinger 2002: 1428, vgl. Lehr 2001: 63). Zugleich werden Auswirkungen der Berichterstattung über Gewaltkriminalität insbesondere auf eine strafverschärfende Kriminalpolitik diskutiert (Windzio, Simonson, Pfeiffer und Kleimann 2007: 7-8, Pfeiffer, Kleimann und Windzio 2004: 415- 435, Pfeiffer 2005, Rückert 2003, Leutheusser-Schnarrenberger 2000, Walter 1994, 1999). In diesem Zusammenhang bleibt allerdings offen, inwieweit Journalistinnen und Jour- 
nalisten abseits einer Publikumsorientierung eigensinnig politische Wirkungen beabsichtigen oder ob diese Effekte als Nebenprodukt einer Erfüllung vermuteter Publikumsbedürfnisse eintreten.

Die Kommunikatorforschung hat sich der journalistischen Motive, über Gewaltkriminalität $\mathrm{zu}$ berichten, bislang kaum angenommen. Lediglich auf vereinzelte Debatten im Journalismus ist zurückzugreifen. Mit der Einführung des Privatfernsehens in Deutschland und neuen Sendeformaten mit einer stärkeren Berichterstattung über Gewalt kommt es zu intensiven Diskussionen über Gewalt im Fernsehen. So meint Ernst Elitz, seinerzeit Fernsehchef des Süddeutschen Rundfunks, dass Gewaltberichterstattung für ein großes Publikum attraktiv ist. Daher erwartet er, dass sie von markt- und renditeorientierten Fernsehsendern stärker betont werden wird und sieht damit zugleich die öffentlich-rechtlichen Sendeanstalten unter Druck (Elitz 1986: 9). Peter Voß, damals Leiter der ZDF-Hauptredaktion „Aktuelles“, zeigt sich ebenfalls von der starken, vor allem emotionalen Wirkung von Gewaltberichterstattung auf das Publikum überzeugt. Doch werde man nicht der Versuchung nachgehen, diese Wirkung auszubeuten (Voß 1988: 147, vgl. Voß 2008: 11). Günter Struve, ehemals Programmdirektor des Ersten Deutschen Fernsehens und Vorsitzender der 1993 gegründeten Anti-Gewalt-Kommission der ARD, beschreibt Gewaltberichterstattung als publikumsaffin und rügt die „bedenkenlose Inflationierung von Gewaltdarstellungen (...), die mit dem Kampf des Kommerz-TVs um Einschaltquoten und Marktanteile zu Beginn der neunziger Jahre eskalierte " (Struve 1998: 79).

Die These, dass Gewaltberichterstattung an sich ein Publikumsmagnet ist und dass die Programmverantwortlichen sie deshalb verstärkt aufgreifen, wird vielfach erhoben. Kerbel, US-amerikanischer Fernsehjournalist und Dozent, vertritt die These ,if it bleeds, it leads“ in einem gleichnamigen Buch (Kerbel 2000, vgl. Gross 2006: 4, Karl 2006: 66, Kapuscinski 1999, McManus 1994). Dies wird in besonderem Maße für Sexualverbrechen angenommen (Friedrichsen 2004: 199, vgl. Friedrichsen 2002, Roithmeier 1994: 100). Das DeutschlandRadio vermeldet: „,Rotlicht und Blaulicht gehen immer' ist eine Faustregel in Fernsehredaktionen bei Privaten und Öffentlich-Rechtlichen.“ (Mayr 2006) Empirisch abgesichert sind diese Thesen allerdings nicht.

So wird im deutschen Fernsehjournalismus angezweifelt, dass Gewalt per se und dauerhaft ein großes Publikum anzieht. Dieter Lesche, ehemals RTL-Chefredakteur, hält das Experiment für gescheitert, ein Massenpublikum mit gewaltlastigen Nachrichten zu gewinnen. „Der Versuch ging gründlich daneben, kaum jemand wollte Crime- und Katastrophennews sehen“" (Lesche 2001: 46, vgl. Deppendorf in Koelbl 2001: 81, Mast 1999: 277, Patterson 2000, Ludwig und Pruys 1998: 586f.). Muckenhaupt sieht in „kassandrischen Schlagzeilen“ nur eine Variante der Nachrichtenvermarktung (Muckenhaupt 1998: 123). Zu einer Neubewertung der Attraktivität von Gewalt in nonfiktionalen Formaten kommt Hans Wolfgang Friede, 1998 für die beiden Boulevardmagazine des Privatsenders ProSieben verantwortlich, und er folgert daraus: „Die Zeiten, wo ein Boulevardmagazin ausschließlich aus Blut, Eiter und Sperma bestand, die sind längst vorbei“ (Niedersächsische Landesmedien- 
anstalt 1999: 94). Den Zweifel an einer durchgängig hohen Attraktivität von Gewalt bekräftigen Ruhrmann und Göbbel, die mit einer Online-Befragung Journalistinnen und Journalisten verschiedene Nachrichtenfaktoren bewerten ließen. Unter 22 vorgegebenen Merkmalen der Berichterstattung erreicht der von Ruhrmann und Göbbel als „Gewalt/Aggression“ bezeichnete Nachrichtenfaktor, definiert durch Androhung oder Anwendung von Gewalt, nur den 13. Rang (Ruhrmann und Göbbel 2007: 40-42).

\subsection{Emotionalisierung des Publikums durch Gewaltberichterstattung}

Aus journalistischer Sicht, soweit sie sich aus fragmentarischen Erkenntnissen erschließt, wird das Publikumsinteresse an der Berichterstattung über Gewaltkriminalität vor allem aus Emotionen gespeist. Die Gerichtsreporterin Gisela Friedrichsen führt eine zunehmende Emotionalisierung der Kriminalberichterstattung im Fernsehen auf den verschärften Wettbewerb um die Publikumsgunst zurück (Friedrichsen 2004: 200, Friedrichsen 2002). Krüger sieht „Affektaufladung und Emotionalität“ als Ergebnis einer verstärkten Marktanpassung und daher vor allem als Nachrichtenphilosophie der privaten Fernsehsender (Krüger 2006: 58, vgl. Pfeiffer 2004). Doch auf welche Emotionen Medienverantwortliche mit der Gewaltberichterstattung zielen, ist kaum erforscht. Wegener resümiert in einer Studie zu Reality-TV, dass die Analyse einer journalistischen Strategie, die vor allem durch Emotionalisierung ein möglichst breites Publikum ansprechen will, bislang ausgeblieben ist (Wegener 1994: 47).

Hoffmann entlehnt aus der Poetik von Aristoteles den Gedanken, die Berichterstattung über Kriminalität errege ,wie die klassische Tragödie Furcht und Mitleid, sie handelt vom Scheitern menschlicher Existenz, vom Archaischen der körperlichen Gewalt, vom Leiden der Opfer, vom Einbruch des Irrationalen in die Alltagsrationalität“ (Hoffmann 1992: 58). ${ }^{5}$ Auch wenn vor allem Furcht als Begleitumstand und Folge medialer Gewalt betrachtet wird, ist Empathie als emotionales Echo gleichsam belegt, beispielsweise mit einer standardisierten Publikumsbefragung zur Rezeption von Gewaltszenen des Fernsehens (Früh 2001: 145-147). Voß (2002) zufolge ist bei besonders schockierenden Gewaltereignissen das Empfinden von Bedrohung eng verknüpft mit Empfindungen wie Trauer und Mitgefühl. Medienpsychologische Untersuchungen beschreiben Empathie als Voraussetzung von Furcht, aber auch als eigenständige Folge. Darstellungen von Gefahr und Leid lösen, so vermutet Zillmann, sowohl Furcht um sich selbst wie Empathie mit anderen aus (Zillmann 2004: 119). Daher wird im Folgenden zu fragen sein, welche Rolle erwartete emotionale Reaktionen wie Furcht oder Empathie im journalistischen Entscheidungsprozess spielen. Begriffen eleos und phobos, die lange Zeit als Mitleid und Furcht übersetzt, neuerer Deutung zufolge als Jammer und Schaudern verstanden werden. (Hügli, Anton und Poul Lübcke (Hrsg.) (1991): Philosophielexikon. Personen und Begriffe der abendländischen Philosophie von der Antike bis zur Gegenwart. Reinbek: Rowohlt, S. 315-316.) 


\subsubsection{Bedeutung von Furcht}

Als emotionale Wirkung von Berichterstattung über Gewaltkriminalität wird vor allem Furcht fokussiert (Cantor 2003: 213). Cavender und Bond-Maupin (1993: 307) halten Kriminalität für das ideale Thema, um die Angst großstädtischen Lebens und das damit zusammenhängende Gefühl von Gefahr auszudrücken. Ruhrmann und andere rechnen Kriminalitätsthemen pauschal zu den „Angstthemen“ (Ruhrmann 2005, Maier, Ruhrmann und Klietsch 2006: 26, Ruhrmann und Göbbel 2007: 65, vgl. Früh 2001: 142f., Smaus 1978: 193).

Altheide betrachtet als wesentliche Funktion der Massenmedien, gleichsam in Fließbandproduktion Furcht zu erregen (Altheide 2002: 23, vgl. Altheide und andere 2001, Bolz 2006). Nach einer Inhaltsanalyse von Printtexten und Fernsehbeiträgen wurde das Wort „fear“ in Meldungen und Berichten der Los Angeles Times und in den Hauptnachrichten des US-Senders ABC am häufigsten im Kontext von Verbrechen verwendet (Altheide 2002: 70). Kerbel referiert Debatten im US-amerikanischen Nachrichtenjournalismus, wonach vor allem Professionelle in TV-Lokalnachrichten es für am günstigsten halten, mit der Berichterstattung über Gewaltkriminalität Furcht $\mathrm{zu}$ erregen und damit eine anhaltende Sehbeteiligung zu sichern. Furcht gilt als menschliche Primärreaktion, die im Extremfall die Zuschauenden so fesselt, dass sie das Programm nicht mehr wechseln können (Kerbel 2000: 106) Für die Bedeutung von Furcht für ein Publikumsinteresse an der Berichterstattung über Gewaltkriminalität werden drei Gründe genannt: a) Furcht weckt in besonderer Weise Aufmerksamkeit, b) Furcht stärkt den sozialen Zusammenhalt und c) Furcht eignet sich in besonderer Weise, um Spannung aufzubauen und sie dann wieder aufzulösen:

1. Furcht weckt Aufmerksamkeit: Voß begreift die besondere Aufmerksamkeit für das Besorgniserregende als Wesensmerkmal des Menschen. Danach richtet sich die menschliche Wahrnehmung nicht auf das Gewohnte, sondern auf das, was Risiko und Gefahr signalisiert und vom Gewohnten gerade abhebt (Voß 2008: 11). Kepplinger hält eine generell höhere Aufmerksamkeit für entwicklungsgeschichtlich bedingt: „Für das Überleben ist es wichtiger, einen bedrohlichen Feind zu entdecken als eine willkommene Beute zu erspähen“ (Kepplinger 1998b: 199, vgl. Schulz 1976: 121).

2. Furcht stärkt sozialen Zusammenhalt: Das Modell der sozialen Kohäsion in der Gemeinschaft der Fürchtenden wird in verschiedenen Kontexten vertreten, sei es allgemein als Folge einer Berichterstattung über Verbrechensopfer (Baumann 1995: 10-13) oder spezieller, wenn etwa über die so genannte Russenmafia berichtet wird (Paoli 2004: 304). In der zunehmenden Thematisierung von Furcht sieht Altheide einen Reflex auf gesellschaftliche Veränderungen - eine zunehmend fragmentierte Gesellschaft wird ihm zufolge durch kollektive Furcht verbunden. Danach können Massenmedien ein großes Publikum erreichen, wenn sie auf die verbindende Wirkung der Furcht setzen (Altheide 2002: 153f.). 
3. Furcht eignet sich dazu, Spannung aufzubauen und wieder aufzulösen: Schließlich tragen Massenmedien zur Integration von Störungen bei. Bolz verwendet das Begriffspaar „Irritation und Stabilisierung“ (Bolz 2006, vgl. Luhmann 1996: 44). Insofern ist die Steigerung von Furcht nur ein mögliches Anliegen von Kriminalitätsberichterstattung. Daneben wird die Bewältigung von Furcht sowohl als gesellschaftliche wie individuelle Abwehr diskutiert (Liska und Baccaglini 1990: 360-374). Hickethier spricht von der psychologisch stabilisierenden Wirkung von Gewalt, wenn sie im letztlich erwartbaren Rahmen beispielsweise einer Nachrichtensendung präsentiert und damit „,domestiziert und entschärft“ wird (Hickethier 2002: 675, vgl. Keppler 2006: 262) Dies wird vor allem dann angenommen, wenn die Ergreifung der Schuldigen die Bewältigung der Irritation symbolisiert (Oliver 2003: 93). Diese Auflösung des Bedrohlichen gehört daher zu den wesentlichen Elementen der „Heldenreise“, die als dramaturgisches Konzept fiktionaler wie dokumentarischer Medienformate auf Campbell (2005) und Vogler (2004) zurückgeht.

\subsubsection{Bedeutung von Empathie}

Soweit Empathie als emotionale Folge von Kriminalitätsberichterstattung angenommen wird, ist zu beachten, wie unterschiedlich dieser Begriff verstanden wird. Davis spricht von einem facettenreichen Phänomen und resümiert 15 Jahre eigener Forschung: Danach ist zu Empathie wissenschaftlich so schwer Zugang zu bekommen, weil es so viele Zugänge gibt (Davis 1996: IX). Empathie wird sowohl kognitiv, also als Verstehen von Gefühlen, wie affektiv, also als Mitfühlen, beschrieben.

Von einem eher kognitiven Begriff sind Kunczik und Zipfel geleitet, wenn sie Empathie definieren als „die Fähigkeit, sich in die Rolle anderer hineinzuversetzen“ (Kunczik und Zipfel 2004: 182). Von einem ähnlichen Verständnis geht Baumann aus, wenn er fordert, durch akkurate Information über die Situation des Opfers und institutionelle Reaktionen Empathie für Verhaltensweisen und Reaktionen von Opfern zu entwickeln (Baumann 1995: 177).

Einen eher affektiven Begriff verwendet Batson. Er skizziert einen Spiegeleffekt und nennt Empathie ,an other-oriented emotional response congruent with the perceived welfare of the other" (Batson 1991, Batson und Moran 1999: 911, vgl. Charlton 2004: 133, Hakansson 2006). Mit dem Fokus auf die emotionale Seite der Empathiebildung bezieht sich Bolz auf die Fernsehberichterstattung über Leid und Gewalterfahrung. Indem die Opfer gezeigt werden, erregen sie Mitgefühl, und durch diese Emotionalität wird ihre Situation nachvollziehbar (Bolz 2006). Eilders stellt Empathie in einen Zusammenhang mit Projektion und vermutet dahinter das Publikumsbedürfnis nach positiver Identifikation (Eilders 1996: 8, vgl. Crone 2005: 48f., Maletzke 1998: 76). Für die Bedeutung von Empathie für ein Publikumsinteresse an der Berichterstattung über Gewaltkriminalität werden zwei Gründe angeführt: a) Empathie verstärkt Furcht und b) Empathie schafft - wie die Furcht (s.o.) - sozialen Zusammenhang: 
1. Empathie verstärkt Furcht: Garland beschreibt die Empathie - in aller Regel mit dem Verbrechensopfer - als emotionales Bindeglied zur Furcht des Publikums. „Publicized images of actual victims serve as the personalized, real-life, it-could-beyou metonym for a problem of security that has become a defining feature of contemporary culture“ (Garland 2002: 11). Katz spricht vom „empathischen Schock", der die eigene Verletzlichkeit vor Augen führt (Katz 1987: 63, vgl. Zillmann 2004: 119). Es erscheint zwingend, dass die Berichterstattung den Opfern Gestalt gibt, damit sie als Projektionsfläche von Empathie dienen können (vgl. Grimm 1999). Damit ist die Frage verknüpft, inwieweit die mediale Aufmerksamkeit den Opfern gilt und inwiefern Medienschaffende ein besonderes - womöglich empathisches - Interesse ihres Publikums für die Leidenden vermuten und daraus Publikationsentscheidungen ableiten.

2. Empathie verbindet: Friedrichsen beschreibt die soziale Kohäsion durch die kollektive Empathie, die ihr zufolge vor allem die Berichterstattung über Verbrechen an Kindern auslöst. So sehr sich unsere Gesellschaft in zahlreiche unterschiedliche Lebenswelten zersplittert, so wenig Einigkeit es über gesellschaftliche Normen gibt - in diesem Punkt herrscht überwältigendes Einverständnigs. „Der Bedarf nach diesem Gefühl von Zusammengehörigkeit - im ,Aufstand der Anständigen' und ähnlichen Stimmung machenden Formeln - findet er seine Befriedigung“ (Friedrichsen 2004: 199, vgl. Paoli 2004: 304).

\subsubsection{Zusammenfassung}

Die These, dass Gewaltberichterstattung an sich ein starkes Publikumsinteresse weckt und Programmverantwortliche sie deshalb verstärkt aufgreifen, wird vielfach erhoben. Anfang der 1990er Jahre ziehen sich in der Bundesrepublik vor allem die privaten Fernsehsender den Vorwurf zu, mediale Gewalt einzusetzen, um ein großes Publikum zu gewinnen. Dagegen äußern Programmverantwortliche Zweifel daran, dass Gewalt per se geeignet ist, um hohe Einschaltquoten zu erzielen.

Nach journalistischer Vermutung ist die Berichterstattung über Gewaltkriminalität aber besonders dazu geeignet, das Publikum emotional zu erreichen und zu binden. Im Vordergrund steht die Annahme, dass die Berichterstattung Furcht weckt. Die Bedeutung der Furcht wird damit begründet, dass sie Aufmerksamkeit weckt, den sozialen Zusammenhalt stärkt und dazu geeignet ist, zunächst Spannung aufzubauen, um daraus Erleichterung zu verschaffen. Auch Empathie wird als eine für das Publikum zentrale Emotion beschrieben. Sie kann sowohl dazu dienen, Furcht zu schüren, als auch ein Gefühl von Zusammengehörigkeit zu schaffen. Damit ist zu fragen, wie im derzeitigen Fernsehjournalismus der Stellenwert von Gewaltkriminalität mit Blick auf das Publikumsinteresse bewertet wird und welche Emotionen als Schlüssel zum Publikumserfolg gelten. 


\subsection{Die Nachrichtenwerttheorie und die Fernsehberichterstattung über}

\section{Gewaltkriminalität}

Im Folgenden wird der theoretische Ertrag der Nachrichtenwerttheorie mit Blick auf jene Nachrichtenfaktoren betrachtet, die für die Fernsehberichterstattung über Gewaltkriminalität von besonderer Bedeutung sind.

\subsubsection{Kriminalität als eigenständiger Nachrichtenfaktor}

Galtung und Ruge (1965: 70-77) beschreiben Negativismus (,reference to something negative“) als einen von zwölf Nachrichtenfaktoren, worunter sie Zerstörung im weitesten Sinne verstehen, seien es ein Mord oder auch ein Verkehrsunfall oder ein Hausbrand (vgl. Östgaard 1965: 55). Für die Bevorzugung des Negativen, die sie für kulturabhängig halten, nennen sie vier Gründe:

1. Kurze Ereignisdauer: Ihnen zufolge ist das Negative - etwa ein Unfall - ein kurzes Ereignis, während das Aufwachsen eines Menschen bis zu diesem Einschnitt ein langer Prozess ist.

2. Eindeutige Reaktionen: Schlechte Nachrichten vereinen Menschen eher in Ablehnung.

3. Bedürfnis vieler Menschen: Galtung und Ruge gehen davon aus, dass negative Nachrichten latente oder manifeste Bedürfnisse vieler erfüllen.

4. Überraschung: Negatives bricht mit Erwartungen eher als Positives (Galtung und Ruge 1965: 69f., vgl. Althoff 1999: 491).

Diese Begründungen verdeutlichen, wie wenig es dem Entwurf von Galtung und Ruge gerecht wird, die von ihnen definierten Nachrichtenfaktoren einzig als Ereignismerkmale aufzufassen und damit ihren Bezug zur Wahrnehmungspsychologie (,psychology of perception“) auszublenden. Lediglich die erste Begründung ist ereignisbezogen, die weiteren drei Begründungen verweisen auf das Publikum und argumentieren über dessen Bedürfnisse und Erwartungen, die nach dem Verständnis von Galtung und Ruge journalistische Auswahlentscheidungen beeinflussen (1965: 68).

Schulz definiert unter dem Oberbegriff der Valenz einen eigenständigen Nachrichtenfaktor Kriminalität. Diesen stuft er in vier Intensitätsstufen ab, vom geringsten Ereignis ohne jeden Regelverstoß bis zu lebensbedrohlichen Verbrechen (Schulz 1976: 44). Dabei bewegt er sich im kulturellen Kontext seiner Zeit, was besonders an der Einordnung der Abtreibung auffällt - sie gilt wie sexuelle Gewalt und Raub als Verbrechen der zweithöchsten Intensitätsstufe (Schulz 1976: 137).

Staab (1990) nimmt den Nachrichtenfaktor Kriminalität unter dem Begriff Aggression auf, worunter er die „Androhung oder Anwendung von Gewalt mit dem Ziel der Schädigung“" versteht. Damit erstreckt sich der Begriff nicht nur auf tatsächliches, sondern auch auf angedrohtes Geschehen. Den Nachrichtenfaktor Aggression hält er für konsensbedingt und nicht für objektivierbar (Staab 1990: 121f.). Kaum konsensfähig aber dürfte allerdings sein, sowohl die Gewalt gegen 
Sachen als auch die Geiselnahme als ,geringe Aggression“ einzuordnen (Staab 1990: 222). Umso erstaunlicher ist es, dass Maier, Ruhrmann und Klietsch (2006: 73) diese Dimensionierung des Nachrichtenfaktors Aggression übernehmen.

\subsubsection{Bedeutung von Folgenschwere}

Thematisiert wird nun, wie stark die Kriminalitätsberichterstattung vom Ausmaß der tatsächlichen oder mutmaßlichen Folgen von Delikten geleitet ist. Galtung und Ruge (1965: 70-77) definieren Negativismus allgemein als Zerstörung, ohne verschiedene Folgen in ihrer Schwere zu differenzieren. Mit seiner Modellierung von vier Intensitätsstufen des Nachrichtenfaktors Kriminalität impliziert Schulz Folgenschwere als Gradmesser für Kriminalität. Danach gelten Straftaten gegen das Leben als besonders intensive Ausprägung von Kriminalität (Schulz (1976: 44, 137). Ähnlich differenziert Staab (1990: 221f.) den Nachrichtenfaktor Aggression nach der Schwere der Folgen, wobei er Eigentumsschäden und nichttödliche Gewalt als geringe Aggression ansieht, Mord und Unruhen als höhere Intensität sowie Krieg und Bürgerkrieg als höchste Intensität. Ruhrmann und Göbbel (2007) orientieren sich weitgehend an Staabs Entwurf.

Weitere Nachrichtenfaktoren sind auf Gewaltkriminalität zu beziehen - Schulz (1976: 137) führt den Nachrichtenfaktor Schaden ein, der Sachschäden geringer, Personenschäden höher bewertet, wobei sich beide Kategorien überschneiden. Staab (1990) übernimmt den Nachrichtenfaktor Schaden/Misserfolg und ergänzt als weiteren Nachrichtenfaktor möglichen Schaden/Misserfolg. Noch deutlicher definiert er den Verlust von Eigentum als geringen Schaden gegenüber der Verletzung von Menschen als großen und der Zerstörung menschlichen Lebens als größten Schaden. Die Art der Verletzung wird nicht weiter spezifiziert (Staab (1990: 224). Maier, Ruhrmann und Klietsch definieren Schaden sehr allgemein als explizit dargestellte negative Folgen, die sich auf ,materielle, ideelle und existenzielle Sachverhalte beziehen“" (Maier, Ruhrmann und Klietsch 2006: 17).

\subsubsection{Bedeutung von Reichweite}

Untersucht werden soll, wie bedeutsam es für die Berichterstattung über Gewaltkriminalität ist, wie viele Menschen von den berichteten Delikten betroffen sind. Den Begriff der Reichweite als der Betroffenheit eines möglichst großen Publikums fassen von Galtung und Ruge (1965) nur vage unter dem Begriff der Relevanz (,relevance"). Schulz konkretisiert ihn mit dem gleichen Begriff als Nachrichtenfaktor, der in höchster Ausprägung zutrifft, wenn ein ,sehr großer Kreis von Betroffenen“" festzustellen ist (Schulz 1976: 42). In seine Beschreibung eines Nachrichtenfaktors Kriminalität bezieht Schulz Reichweite nicht explizit ein, er spricht hier ausschließlich vom Einzelfall. Dagegen führt Staab (1990: 220) die Reichweite als Dimension des Nachrichtenfaktors Aggression ein, indem er als höchste Intensität 
beschreibt: „größte Aggression: Krieg, Bürgerkrieg“. Damit wird also nicht allein die individuelle Folgenschwere erfasst, sondern auch das Maß, wie viele Menschen gravierende Folgen erleiden. Maier, Ruhrmann und Klietsch (2006: 73) übernehmen diese Definition und ergänzen den Begriff Terror.

Staab umreißt den eigenen Nachrichtenfaktor Reichweite (der den Begriff der Relevanz bei Schulz 1976 ersetzt) mit der Anzahl der Personen, „die an einem Ereignis teilnehmen oder direkt von ihm betroffen sind“" (Staab 1990: 121). Ruhrmann und Göbbel übernehmen die Definition von Staab, allerdings mit einem wesentlichen Unterschied: Sie führen eine subjektive Komponente als Teil der Reichweite ein, die erwartete oder annehmbare Betroffenheit: „Unter der Reichweite eines Ereignisses wird die Anzahl der Personen verstanden, die direkt von ihm betroffen sind / sein werden / waren / sein können“ (Ruhrmann und Göbbel 2007: 41). Damit rückt neben den Begriff der messbaren Reichweite der Begriff der gefühlten Reichweite, der sich nicht auf empirische Gesetzmäßigkeiten bezieht, sondern auf Gefühle und Ahnungen (Kania 2000: 78-97, Walter 2000: 18f.).

\subsubsection{Bedeutung von Personalisierung}

Im Folgenden geht es um die Verdichtung eines Geschehens auf einzelne Personen und ihre Bedeutung als Nachrichtenfaktor. Galtung und Ruge definieren Personalisierung (,personification“) als einen von vier Nachrichtenfaktoren, die sie für kulturabhängig halten. „The more the event can be seen in personal terms, as due to the action of specific individuals, the more probable that it will become a news item" (Galtung und Ruge 1965: 68f.). Sie begründen Personalisierung mit einem Bedürfnis nach Bedeutung und damit nach Identifikation. Diese beschreiben sie sowohl als Abgrenzung zu abgelehnten Personen wie als Empathie mit positiv bewerteten Personen.

Schulz (1976) übernimmt diesen Entwurf nahezu unverändert. In weiteren Studien zur Nachrichtenwerttheorie (Staab 1990, Eilders 1997, Maier, Ruhrmann und Klietsch 2006, Ruhrmann und Göbbel 2007) bleibt Personalisierung als Nachrichtenfaktor enthalten. Ruhrmann und Göbbel (2007: 41) kennzeichnen Personalisierung als „Bedeutung, die Einzelpersonen in einem Ereignis zugesprochen wird“ (Ruhrmann und Göbbel 2007: 41).

\subsubsection{Bedeutung von Eindeutigkeit}

An dieser Stelle wird der Nachrichtenwert von Eindeutigkeit beschrieben, die explizit als Nachrichtenfaktor beschrieben wird und vor allem implizit verschiedenen Nachrichtenfaktoren unterliegt. In ihrer Radiometapher skizzieren Galtung und Ruge die Klarheit und Eindeutigkeit eines Signals als zentrale Bedingung, es zu erfassen (Galtung und Ruge 1965: 65). Dabei unterliegt es einerseits einer Übereinkunft („,convention“), ob ein Geräusch als hörenswert oder als Lärm wahrgenommen 
wird und damit einem Publikumsmerkmal. Andererseits, so nehmen Galtung und Ruge an, lassen sich einige Signale eher als andere in bestimmter Weise verstehen, und damit wird ein Ereignismerkmal beschrieben. Daraus entwickeln sie den Nachrichtenfaktor Eindeutigkeit (,unambiguity“). Diese Eindeutigkeit verknüpfen sie vor allem mit negativ bewerteten Ereignissen, die aus ihrer Sicht den Nachrichtenfaktor Negativismus in besonderer Weise erfüllen. Danach erzielen negative Nachrichten eher einhellige Reaktionen (Galtung und Ruge 1965: 69).

Schulz greift den Nachrichtenfaktor Eindeutigkeit nicht mehr explizit auf, impliziert aber, dass sich bestimmte Ereignisse eindeutig als positiv oder negativ fassen lassen. So begreift er die Nachrichtenfaktoren Konflikt, Kriminalität und Schaden unter dem Oberbegriff des Negativismus, dem er den Nachrichtenfaktor Erfolg gegenüberstellt (Schulz 1976: 34). Die Polarität der Nachrichtenfaktoren Schaden und Erfolg zieht sich durch verschiedene Studien in Anlehnung an die Nachrichtenwerttheorie (Staab 1990, Eilders 1997, Ruhrmann, Woelke, Maier und Diehlmann 2003, Maier, Ruhrmann und Klietsch 2006). Dass dieser Kategorisierung eine normative Setzung unterliegt, wird nicht weiter thematisiert. Lediglich Schulz (1976: 137) begründet den Nachrichtenfaktor Kriminalität mit dem Referenzrahmen gültiger Gesetze - damit ist als negativ definiert, was rechtswidrig ist. Staab (1990: 121) leitet die Polarität der Nachrichtenfaktoren Nutzen/Erfolg bzw. Schaden/Misserfolg vage aus „allgemeinem Verständnis“ ab. Maier, Ruhrmann und Klietsch (2006: 17) verzichten gänzlich darauf, die Abgrenzung der Nachrichtenfaktoren Nutzen/Erfolg bzw. Schaden/Misserfolg voneinander zu begründen (vgl. Ruhrmann und Göbbel 2007: 41). Ob aber etwa das Schlagen des eigenen Kindes als nützliche Erziehungsmaßnahme oder schädliche Gewalt, der Tyrannenmord als nützlich oder schädlich zu betrachten ist, lässt sich nur innerhalb eines bestimmten Referenzrahmens, und damit nicht per se beantworten und den vorgegebenen Nachrichtenfaktoren zuordnen. Dabei lässt sich diesen Nachrichtenfaktoren jenseits jeder normativen Festlegung ein Muster unterlegen, das der Eindeutigkeit. Sie beschreibt - im Rückgriff auf den von Galtung und Ruge verwendeten Begriff der „unambiguity" - die Verdichtung in sich stimmiger Eigenschaften.

\subsubsection{Bedeutung von Visualität}

Der Nachrichtenfaktor Visualität gründet sich auf die Verfügbarkeit von Bildmaterial und die Folgen für die Berichterstattung. Galtung und Ruge (1965), die ihren Entwurf der Nachrichtenwerttheorie empirisch auf die Berichterstattung von vier norwegischen Zeitungen stützen, haben Visualität als Nachrichtenfaktor nicht im Blick. Zwar kreist ihr Entwurf wesentlich um das Bild (,image“), das sich die Öffentlichkeit und die Medien von der Wirklichkeit machen, aber Fotografien und bewegte Bilder als Mittel erwähnen sie nicht. Der Vorschlag von Buckalew, Visualität - genauer: „visual availability“ als eigenständigen Nachrichtenfaktor (,news facet“) zu betrachten, setzt sich nicht durch (Buckalew 1969: 135-137). 
Schulz (1976), der sowohl Zeitungen, Hörfunk- und Fernsehnachrichten wie Agenturmeldungen analysiert, damit also auch bewegte Bilder untersucht, schenkt der Bedeutung der Bilder nicht explizit Beachtung. Auch bei Staab (1990) spielt dieser Aspekt keine Rolle. Erst die Forschungsgruppe um Ruhrmann führt den Nachrichtenfaktor Visualität ein (Ruhrmann, Woelke, Maier und Diehlmann 2003).

\subsubsection{Zusammenfassung}

Die Nachrichtenwerttheorie bildet den Referenzrahmen der vorliegenden Studie, um journalistische Auswahl- und Thematisierungsmuster bei der Fernsehberichterstattung über Gewaltkriminalität $\mathrm{zu}$ beschreiben. Galtung und Ruge (1965) sprechen allgemein von Negativismus als Nachrichtenfaktor. Die mediale Bevorzugung des Negativen - beispielsweise eines Mordes als Berichtsthema begründen sie formal damit, dass ein solches Ereignis von kurzer Dauer ist, und mit Blick auf das Publikum damit, dass schlechte Nachrichten Menschen in Ablehnung vereinen, die Bedürfnisse vieler erfüllen und besonders überraschen. Schulz (1976) entwickelt einen Nachrichtenfaktor Kriminalität, den Staab (1990) Aggression nennt, Eilders (1997) unter Kontroverse subsumiert und Ruhrmann, Woelke, Maier und Diehlmann (2003) wieder als Nachrichtenfaktor Kriminalität aufführen.

Weitere Nachrichtenfaktoren sind auf Gewaltkriminalität $\mathrm{zu}$ beziehen Folgenschwere wird explizit definiert durch Nachrichtenfaktoren wie Schaden/ Misserfolg, implizit durch die Dimensionierung beispielsweise des Nachrichtenfaktors Kriminalität, wonach besonders gravierenden Gewalttaten ein höherer Nachrichtenwert zugeschrieben wird (Schulz 1976). Die Betroffenheit möglichst vieler Menschen bezeichnen Galtung und Ruge (1965) und Schulz (1976) als Relevanz, bei Staab (1990) und in späteren Entwürfen wird sie als Reichweite definiert. $\mathrm{Zu}$ unterscheiden sind die messbare Reichweite (wie viele Menschen ein Ereignis tatsächlich betrifft) und die gefühlte Reichweite (wie viele Menschen sich von einem Ereignis betroffen fühlen).

Personalisierung als Nachrichtenfaktor durchzieht die Nachrichtenwerttheorie nahezu unverändert. Der von Galtung und Ruge definierte Nachrichtenwert der Eindeutigkeit wird seit Schulz 1976 aufgegeben. Die Annahme von Eindeutigkeit liegt allerdings zugrunde, wenn beispielsweise Schaden von Nutzen abgegrenzt wird. Als weiterer Nachrichtenfaktor ist Visualität als die Verfügbarkeit von Bildmaterial zu nennen - die Forschungsgruppe um Ruhrmann nimmt diesen Faktor 2003 in den Faktorenkatalog auf.

Diese Faktoren werden nahezu durchweg inhaltsanalytisch vom Ergebnis der Abwägungen in den Medien her beschrieben. Offen bleibt, wie sich Nachrichtenfaktoren im journalistischen Entscheidungsprozess konkret auswirken, wie sie bestimmt und gewichtet werden, wie sie Auswahl- und Thematisierungsmuster prägen und welche Rolle publikumsbezogene Wirkungsannahmen und Wirkungsabsichten dabei spielen. 


\subsection{Empirische Befunde zur Kriminalitätsberichterstattung}

Im Mittelpunkt dieses Kapitels stehen Inhaltsanalysen der Berichterstattung über Kriminalität, insbesondere über Gewaltkriminalität. Wenn den Aussagen von Journalistinnen und Journalisten $\mathrm{zu}$ folgen ist, dann ist medial vermittelte Gewaltkriminalität nicht kategorisch, sondern unter bestimmten Bedingungen dazu geeignet, das Publikum zu emotionalisieren und damit zu gewinnen und zu halten. Die Nachrichtenwerttheorie wurde daraufhin betrachtet, welche Nachrichtenfaktoren für die Berichterstattung über Gewaltkriminalität aus theoretischer Perspektive bedeutsam sind. Im folgenden Kapitel sollen empirische Funde daraufhin analysiert werden, welche Muster der Berichterstattung sich darin zeigen. Damit geht es um die fünfte Leitfrage: Inwieweit sind die zuvor beschriebenen journalistischen Auswahl- und Thematisierungskriterien handlungsrelevant? Dazu werden zunächst Gewalt und Gewaltkriminalität definiert, Befunde zur Berichterstattung über Gewaltkriminalität vorgestellt und anschließend die Medieninhalte nach den aus der Nachrichtenwerttheorie abgeleiteten relevanten Nachrichtenfaktoren analysiert.

\subsubsection{Begriffsklärung}

Untersuchungen zur Gewalt in den Medien unterscheiden sich nicht nur in ihrem jeweiligen Forschungsinteresse, sondern auch in ihrem Begriff von Gewalt. So kann verbrecherische Gewalt mit criminal violence übersetzt werden, tödliche Gewalt mit deadly force, Staatsgewalt mit authority of the state. So vielschichtig also der Begriff von Gewalt ist, wird er im Folgenden nach vier Dimensionen umrissen.

1. Von wem geht Gewalt aus? Eine wesentliche Dimension des Gewaltbegriffs bezieht sich auf die Urheberschaft der Gewalt. Gewalt kann als allein menschliches Handeln bezeichnet werden (Federman 1998: 18, Kunczik und Zipfel 2004: 10, Früh 2001: 39) oder Naturgewalten und technische Defekte einschließen (Grimm, Kirste und Weiß 2005: 64).

2. Ist Gewalt beabsichtigt? Soweit es um von Menschen verursachte Gewalt geht, kann damit ausschließlich absichtsvolles Handeln (Federman u.a. 1997: 41) oder auch die unbeabsichtigte Schädigung gemeint sein (Krüger 1994: 73).

3. Wie äußert sich Gewalt? $\mathrm{Zu}$ unterscheiden sind weiter die Formen der Gewalt. Dabei kann es in einem engeren Verständnis allein um körperliche Schädigung (Federman u.a. 1997: 41) gehen, in der offensten Definition um das „Eintreten von physischem, psychischem, materiellem, sozialem und ökologischem Schaden“ (Krüger 1994: 73).

4. In welchem Zusammenhang steht Gewalt? Eine Reihe von Studien zur Gewalt im Fernsehen befasst sich mit Gewalt einschließlich kriegerischer Handlungen (vgl. 
Grimm, Kirste und Weiß 2005, Winterhoff-Spurk, Unz und Schwab 2005, Mast 1999, Groebel und Gleich 1993), während andere Studien auf Kriminalität fokussieren (vgl. Cavender und Fishman 1998, Morris 1997, Fishman 1978).

Wenn bereits das Eigentumsdelikt, mit dem Menschen lediglich materiell geschädigt werden, oder das Naturereignis, das ökologischen Schaden anrichtet, als Gewalt definiert werden, dann meint Gewalt nahezu alles - vom Verlust eines Koffers bis zum Hagelschauer - und damit nichts. Gewalt wird in dieser Untersuchung verstanden als menschliches Verhalten, das absichtlich oder absehbar Menschen körperlich bzw. seelisch schädigt oder als die Androhung eines solchen Verhaltens. Das Forschungsinteresse gilt berichteter Gewalt im Inland.

Mit diesem Verständnis von Gewalt wird der Begriff der Kriminalität verbunden - er geht zurück auf das lateinische crimen, das sowohl das Verbrechen wie auch Schuld und Vorwurf bezeichnet. Damit rückt neben den äußeren Merkmalen einer Tat auch der mit ihr zusammenhängende Regelbruch in den Blick. Als Regelwerk wird das deutsche Strafgesetzbuch zu Grunde gelegt. Dabei spielt allerdings keine Rolle, ob eine Gewalttat strafrechtlich verfolgt werden kann oder nicht, beispielsweise weil ein Tatverdächtiger strafunmündig ist. Übereinstimmend mit der Definition der Polizeilichen Kriminalstatistik (BKA 2008: 16, Summenschlüssel 8920) werden folgende Straftaten als Gewaltkriminalität definiert: Mord, Totschlag und Tötung auf Verlangen, Vergewaltigung und sexuelle Nötigung, Raub, räuberische Erpressung und räuberischer Angriff auf Kraftfahrer, Körperverletzung mit Todesfolge, gefährliche und schwere Körperverletzung, erpresserischer Menschenraub, Geiselnahme und Angriff auf den Luft- und Seeverkehr. Als Gewaltkriminalität werden nach dem oben ausgeführten Verständnis von Gewalt weitere Delikte einbezogen: sämtliche Straftaten gegen die sexuelle Selbstbestimmung, beispielsweise der sexuelle Missbrauch, alle Körperverletzungsdelikte einschließlich der Misshandlung Schutzbefohlener und alle Straftaten gegen die persönliche Freiheit, etwa Stalking. Umfasst werden allgemeine Formen der Thematisierung und die Berichterstattung über einzelne Gewalttaten wie auch deren Entstehung, Verfolgung, Aufklärung und Folgen.

\subsubsection{Allgemeine Befunde}

Das Forschungsinteresse gilt der berichteten Gesamtkriminalität - soweit Studien die Berichterstattung über Kriminalität im Allgemeinen beschreiben, werden sie im Folgenden auf das engere Forschungsinteresse hin fokussiert.

Kriminalitätsberichterstattung ist ein altes Phänomen

$\mathrm{Zu}$ frühen Beispielen der Kriminalitätsberichterstattung gehört die „Erweiterte Unholdenzeitung“, eine illustrierte Flugschrift zu Verbrechen und deren Bestrafung aus dem Jahre 1590 (Höbermann 1989: 13). Stieler referiert 1695, dass Zeitungen jener Zeit Ereignisse aufgreifen wie „Ehebruch / Hurerey / Kindermord / Diebstal / 
Todschlag / Verräterey / und wie diß alles künstlich volbracht worden und vertuschet werden wollen“ (Stieler 1969: 61). Der Dichter Friedrich Schiller verfasst von 1792 bis 1795 Reportagen über außergewöhnliche Rechtsfälle und zeigt sich überzeugt: „In der ganzen Geschichte der Menschen ist kein Kapitel unterrichtender für Herz und Geist, als die Annalen seiner Verirrungen“ (Tekolf 2005: 7). Mit seinem Anspruch aufklärerischer Berichterstattung grenzt er sich ab von ,gewinnsüchtigen Verlegern“, die aus dem Grauen lediglich Profit ziehen wollten (ebd.: 75). Systematische Untersuchungen der Kriminalitätsberichterstattung sind seit Ende des 19. Jahrhunderts bekannt. So sieht Speed in New Yorker Zeitungen eine auffällige Neigung zum Schrecklichen: Danach berichten die Zeitungen nicht über die wirklich wichtigen Ereignisse, sondern über die Sensationen und Katastrophen der Geschichte (Speed 1893: 710, nach Schulz 1989: 135, vgl. Garofalo 1981: 321, Fenton 1910, Swanson 1955: 416f.).

Kriminalitätsberichterstattung schwankt in ihrem Ausmaß im Zeitverlauf

Vielfach wird die Ansicht vertreten, Gewaltkriminalität errege per se ein hohes Publikumsinteresse und sei darum kategorisch ein Medienthema ersten Ranges (Egg 2003: 115, Dulinski 2003: 73, Kerbel 2000, Gross 2006: 4, Karl 2006: 66, Kapuszinski 1999, McManus 1994, Walter 2000: 14, Walter 1999: 350). Diese kategorische Annahme lässt sich nicht stützen. Nach Wilke, der Zeitungen aus vier Jahrhunderten analysiert, hat die Berichterstattung über Kriminalität erst in neuerer Zeit an Bedeutung gewonnen (Wilke 1984: 162-164). Westerstahl und Johansson (1986) erforschen im Langzeitvergleich schwedischer Medien zwischen 1912 und 1984 die Gewichtung von Ereignissen, die sie als Negativismus fassen. Danach verlieren in den 70er Jahren Themen wie Kriminalität gegenüber Umweltschäden und sozialen Entwicklungen an Bedeutung (Westerstahl und Johansson 1986, vgl. Kepplinger und Weissbecker 1991). Neuere Befunde zum deutschen Fernsehen deuten auf eine wieder stärkere Gewichtung von Kriminalität hin. Maier, Ruhrmann und Klietsch stellen für die deutschen Fernsehnachrichten zwischen 1992 und 2004 einen mehr als verdoppelten Umfang der „Angstthemen“ wie Kriminalität, Unfälle und Katastrophen fest, von 9 Prozent im Jahr 1992 auf 21 Prozent im Jahr 2004 (Maier, Ruhrmann und Klietsch 2006: 46-49). Diese gewachsene Publikumsorientierung im Journalismus hat, so resümiert eine Reihe von Studien, Folgen für die Kriminalitätsberichterstattung. Sie ist Löhr zufolge immer stärker an den Gesetzen von Markt und Konkurrenz orientiert (Löhr 2003: 541, vgl. Bielstein 1996: 1042, Chibnall 1981: 75). Pfeiffer, Windzio und Kleimann kritisieren, dass die Darstellung von Gewaltkriminalität zunehmend von den erwarteten Zuschauerquoten diktiert wird (Pfeiffer, Windzio und Kleimann 2005, vgl. Windzio und Kleimann 2006: 196, Windzio, Simonson, Pfeiffer und Kleimann 2007: 6, Kepplinger 2002: 1428, Norek 2005: 20, Groebel und Gleich 1993: 20, Gleich 1995: 156).

Gleichwohl führt eine Publikumsorientierung keineswegs zu einer durchgängig starken Gewichtung des Themas Gewaltkriminalität. So lassen sich auffällige Schwankungen an den Hauptnachrichten der drei größten US-amerikanischen Sen- 
der nachweisen. Zwischen 1958 und 1977 wurden Anteile bis zu einem Drittel an den gesamten Nachrichten gemessen (Garofalo 1981: 325f., vgl. Graber 1980: 24, Gerbner 1970: 74). Nach einer Vollerhebung aller Hauptnachrichten von ABC, CBS und NBC der vergangenen 20 Jahre ist der Anteil nach dem Höchstwert von 16,4 Prozent im Jahre 1995 (mit zahlreichen Berichten über den Mordprozess gegen den Football-Star O. J. Simpson) auf 1,8 Prozent im Jahre 2008 gefallen $^{6}$ und liegt damit deutlich unter den Anteilen an den Hauptnachrichten von ARD, ZDF, RTL und Sat.1, die in den Jahren 2005 bis 2007 im Schnitt konstant zwischen 5,2 und 5,3 Prozent betragen (Tyndall 2009, Krüger 2008: 59) und 2008 auf 5,8 Prozent steigen (Krüger 2009: 75).

Abb. 3: Anteil der Kriminalitätsberichterstattung an den Hauptnachrichten der US-Sender ABC, CBS und NBC 1988 - 2008

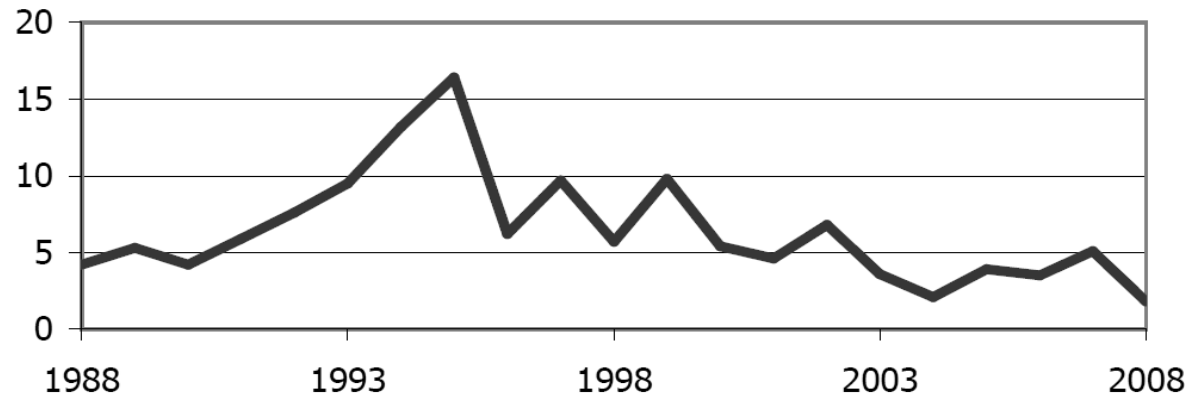

Prozentualer Anteil an der Sendungslänge der Hauptnachrichten der US-amerikanischen Fernsehsender ABC, CBS und NBC, jeweils über das gesamte Jahr. Eigene Grafik nach Tyndall (2009).

Schwankungen der Kriminalitätsberichterstattung sind durch Kriminalitätsstatistiken vielfach nicht zu erklären

Ende des 19. Jahrhunderts wertet der italienische Jurist Lino Ferriani die zunehmende Berichterstattung über 232 Fälle von Kindesmisshandlung aus und versucht zu ergründen, ob es tatsächlich eine Zunahme dieser Verbrechen gegeben hat. Damit

6 Die Kriminalitätsberichterstattung nimmt dagegen in den Lokalnachrichten des USFernsehens einen breiten Raum ein (Project for Excellence in Journalism 2006, Patterson 2000, Graber 1980).

7 Tyndall untersucht die Fernsehnachrichten der drei größten US-Sender in einer Vollerhebung und hat die Daten zur Kriminalitätsberichterstattung zwischen 1988 und 2008 dem Verfasser freundlicherweise zur Verfügung gestellt. Für das deutsche Fernsehen legt das Institut für empirische Medienforschung mit seinem Infomonitor ähnlich exakte Daten zu den Hauptnachrichten von ARD, ZDF, RTL und Sat. 1 erst seit 2005 vor. 
stellt sich schon früh die Frage, ob ein Medienphänomen auf tatsächliche Veränderungen der Kriminalität oder auf veränderte journalistische Auswahlentscheidungen zurückzuführen ist (Ferriani 1897 nach Kaiser 2002: 180, vgl. Roshier 1981, Hestermann 2003: 71).

Wie wenig Medientrends mit amtlichen Statistiken zu erklären sind, weist Fishman (1980) nach. Er analysiert die sprunghafte Zunahme der Berichterstattung über Kriminalität gegen Ältere im Herbst 1976 in New York, während die Zahl der polizeilich gemeldeten Delikte zugleich zurückgeht. Fishman spricht von einer Kriminalitätswelle (,crime wave“), die im Grunde eine Welle des berichteten Verbrechens ist (Fishman 1980: 5, vgl. Cavender und Fishman 1998: 3-15, Fishman 1978: 531543, Vasterman 2005). Eine solche Welle wurde bereits rund 50 Jahre zuvor bekannt - der Reporter Lincoln Steffens schildert, wie er selbst eine Welle von Berichten über Einbrüche gleichfalls in New York ausgelöst hat: „I enjoy crime waves. I made one once" (Steffens 1931: 285).

Eine deutlich veränderte Gewichtung von Kriminalität als Nachrichtenthema jenseits statistischer Logik legt Morris dar. Während zwischen 1991 und 1995 die Gewaltkriminalität in den USA nach polizeilicher Erkenntnis abnimmt, vervierfachen die Hauptnachrichten der drei US-weiten Fernsehsender ABC, CBS und NBC die Zahl ihrer Beiträge über Verbrechen (Morris 1997: 108, vgl. Tyndall 2009).

Unterschiede und Konvergenz zwischen öffentlich-rechtlichen und privaten Sendern

Anfang der 90er Jahre wird die Gewalthaltigkeit des deutschen Fernsehens breit diskutiert, ausgelöst vor allem durch die umfangreiche Thematisierung von Gewalt in neu entstandenen Boulevardmagazinen wie RTL Explosiv sowie weiteren Fernsehformaten des Privatfernsehens, die unter dem Genre „Reality TV“ eingeordnet wurden (Wegener 1994, Kammann 2002, Zachert 1994: 685f.). Die Bundesregierung (1998: 97) rügte ,die etwa seit Ende der achtziger Jahre verstärkt auftretende Ausstrahlung von Gewaltszenen vor allem in den Programmen privater Fernsehveranstalter". Zahlreiche Analysen verweisen seitdem auf systematische Unterschiede zwischen öffentlich-rechtlichen und privaten Fernsehanbietern - beispielsweise Bartel (1997) mit seiner Befragung von 29 leitenden Journalistinnen und Journalisten sowie 40 weiteren Interviews mit dem journalistischen Personal in sechs Fernsehnachrichtenredaktionen. Krüger und Zapf-Schramm (2001: 326-344) ziehen aus ihrer vergleichenden Inhaltsanalyse von vier Programmwochen von ARD, ZDF, RTL, SAT.1 und ProSieben das Fazit, zwischen öffentlich-rechtlichen und privaten Anbietern klaffe eine „Boulevardisierungskluft“ (vgl. Krüger und Zapf-Schramm 2000, 2003, 2006, 2007, Krüger 2005).

Für die Hauptnachrichten der größten Sender sind systematische Unterschiede zwischen öffentlich-rechtlichen und privatrechtlichen Anbietern klar belegt. Nach Krüger behandeln die Nachrichten von RTL und Sat.1 Kriminalitätsthemen anteilig dreimal so umfangreich wie die öffentlich-rechtlichen Nachrichten (Krüger 2008: 59, vgl. Bruns 1998, Bruns und Marcinkowski 1997, Groebel und Gleich 1993: 76, Grimm, Kirste und Weiß 2005). Systematische Unterschiede zwischen den Sender- 
gruppen zeigen sich nicht, wenn Boulevardmagazine untersucht werden. Mikos (1998: 67) stellt sowohl für das öffentlich-rechtliche Magazin Brisant (ARD) wie die privaten Konkurrenten Explosiv (RTL) und taff (ProSieben) Anteile des Themenfelds Kriminalität von über 20 Prozent fest. Krüger und Zapf-Schramm (2003: 543) konstatieren mit 11 Prozent Kriminalitätsberichterstattung etwas höhere Anteile an den öffentlich-rechtlichen Boulevardmagazinen als bei den Formaten des Privatfernsehens mit 10 Prozent.

Augenmerk wird vielfach auf Unterschiede zwischen den Informationsangeboten öffentlich-rechtlicher und privater Fernsehsender gelegt. Prokop geht so weit zu behaupten: „Blutbad-Bilder verkörpern die Corporate Identity der kommerziellen Fernsehkanäle.“ (Prokop 1998: 964) Vergleichende empirische Studien deuten allerdings auf eine Konvergenz insbesondere durch eine Annäherung öffentlichrechtlicher Sendungen an die kommerzielle Konkurrenz hin. Bruns und Marcinkowski stellen zwischen 1986 und 1994 eine besonders ausgeprägte Steigerung von Gewaltbildern bei öffentlich-rechtlichen Informationssendungen fest. „Zumindest hinsichtlich der Gewalt kann die pauschale Unterscheidung öffentlich-rechtlich versus privat-kommerziell nicht aufrechterhalten werden“ (Bruns und Marcinkowski 1997: 227f., vgl. Bruns 1998). Winterhoff-Spurk, Unz und Schwab beobachten gleichfalls eine Annäherung der Gewaltanteile in den Hauptnachrichten zwischen 1996 und 2002 - dabei weisen die privaten Sender im Jahr 2002 noch einen anderthalbfach so hohen Gewaltanteil wie die öffentlich-rechtlichen Sender auf (Winterhoff-Spurk, Unz und Schwab 2005: 225-237).

\subsubsection{Vermittlung von Folgenschwere}

Die theoretisch begründete Fokussierung auf körperliche Gewalt (vgl. Kap. 3.3.2) spiegelt sich in zahlreichen empirischen Befunden wider (Baumann 1995: 28, vgl. Schulz 1976: 82, van der Broeck 1996: 118, Lamnek 1990: 165, Obermöller und Gosch 1995: 52, Grimm, Kirste und Weiß 2005). Mord, Geiselnahme, Kindesmissbrauch und weitere Gewaltdelikte wie Körperverletzung machen 64,8 Prozent der Kriminalitätsberichterstattung der Hauptnachrichten von ARD, ZDF, RTL und Sat.1 aus, wobei die Privatsender stärker gewaltorientiert berichten als die öffentlichrechtlichen Sender (Krüger 2008: 70, siehe Abb. 4).

Im Vergleich verschiedener Gewaltdelikte finden jene das stärkste Medieninteresse, die tödlich enden. International zeigen Inhaltsanalysen, dass drastische Gewalt, vor allem Mord und Totschlag, überproportional berichtet werden (Garofalo 1981: 323). Übereinstimmend ermitteln Studien, dass etwa jedes zweite Gewaltopfer, über das im deutschen Fernsehen berichtet wird, zu Tode gekommen ist (Groebel und Gleich 1993: 102, Grimm, Kirste und Weiß 2005: 120, Krüger 2008: 70, Winterhoff-Spurk 1994: 57). Daraus folgern Groebel und Gleich: „Die Tötung von Menschen ist zum Teil zu einem selbstverständlichen Programmelement geworden“" (Groebel und Gleich 1993: 73). Diese Betonung besonders gravierender Delikte wird vielfach gerügt. Pfeiffer spricht von einer Dämonisierung des Bösen, Bourdieu von der Sensation als Auswahlkriterium (Pfeiffer 2004: 9, Bourdieu 1998: 25). 
Abb. 4: Delikte der Kriminalitätsberichterstattung in deutschen Fernsehnachrichten

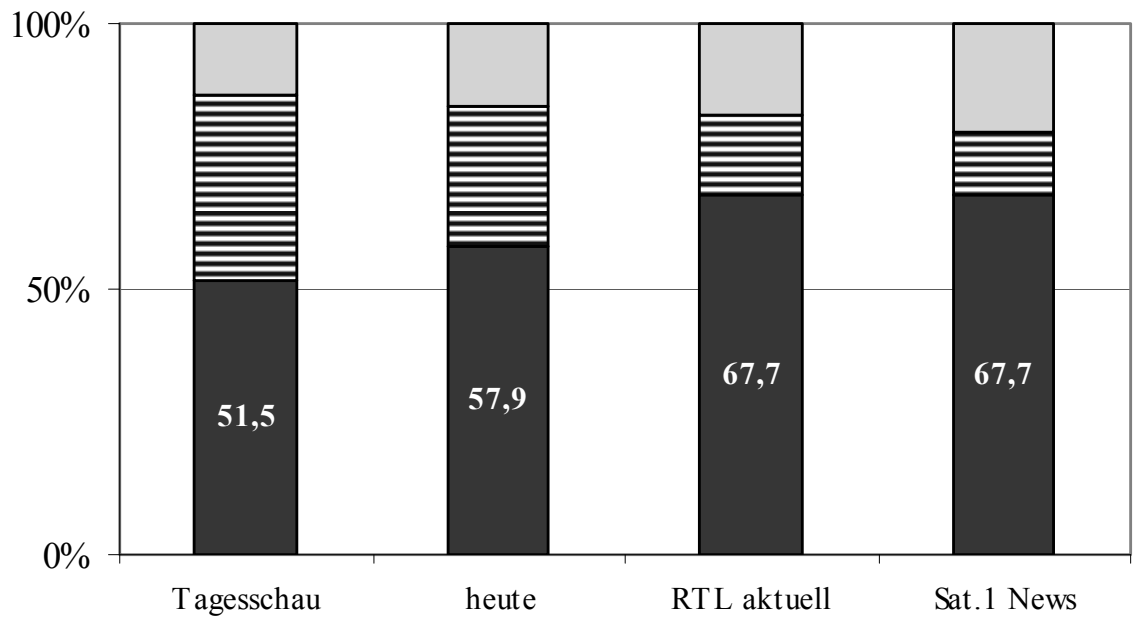

$\square$ Gewalt $\boxminus$ Eigentums- und Drogendelikte $\square$ Sonstige, Verbrechensbekämpfung

Prozentualer Anteil der Kriminalitätsberichterstattung an den Hauptnachrichten von ARD, ZDF, RTL und Sat.1. Untersuchungszeitraum: 1. 1. - 31. 12. 2007. Eigene Grafik nach Krüger (2008: 70).

Neuere Studien nehmen auch die erhöhte Bedeutung sexueller Gewalt für journalistische Auswahlentscheidungen in den Blick. Nach Baurmann wird in den 1970er und 80er Jahren zunächst in den USA und dann in Europa die Wahrnehmung sexueller Gewalt in der Partnerschaft und von sexuellem Missbrauch besonders an Kindern enttabuisiert (Baurmann 2004: 436). Seitdem mehren sich Befunde, dass sexuelle Gewalt besonders intensiv fokussiert wird (Bundesministerium des Inneren und Bundesministerium der Justiz 2006: 60f., Schulenburg 2007: 1, Reuband 1997, Proctor, Badzinski und Johnson 2002: 357).

So liegt nahe, dass über sowohl sexuelle wie tödliche Gewalt zunehmend intensiv berichtet wird. Tatsächlich beobachten Schneider, Arnold und Greve einen starken Anstieg an Berichten über Sexualmorde an Kindern. Sie untersuchen die Berichterstattung zwischen 1971 und 2001 über jeweils zwei Fälle in Zeitungen, Zeitschriften und Nachrichten- und Magazinsendungen des Fernsehens. Zwischen 1971 und 1996 schwanken die Zahlen nur gering zwischen jeweils 5 und 23 Berichten, vor 1991 ausschließlich in Boulevard- und Regionalzeitungen. Die Fernsehberichterstattung setzt 1991 mit vier Berichten des Privatfernsehens ein, 1996 berichtet das Privatfernsehen 17-mal. Das öffentlich-rechtliche Fernsehen ist erstmals 2001 insgesamt 34-mal vertreten (Privatfernsehen 62 Berichte). 2001 explodiert der Umfang 
der Berichterstattung auf 218 Berichte in allen untersuchten Medien, mehr als doppelt so viel wie in den gesamten sechs zuvor beobachteten Jahren (Schneider, Arnold und Greve 2005a).

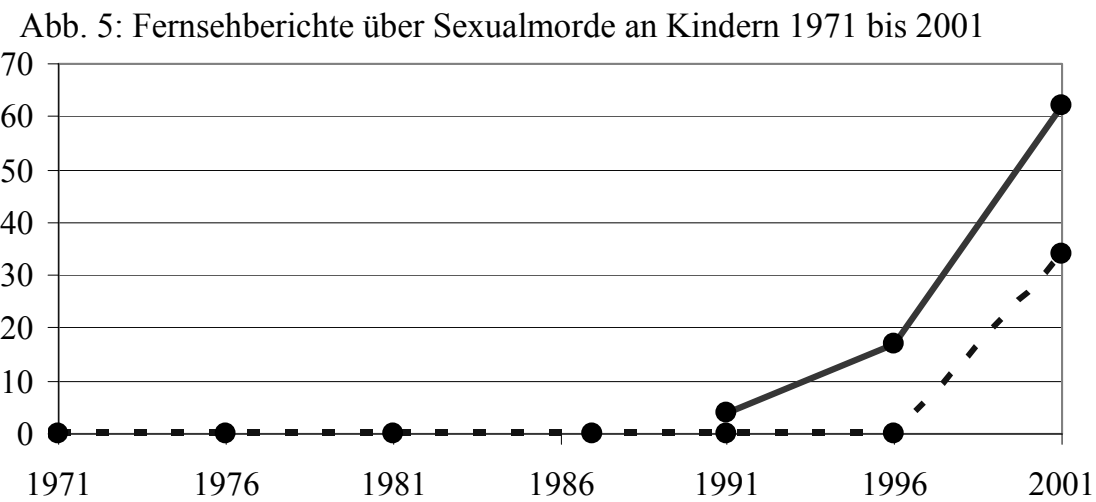

$\longrightarrow$ Privatfernsehen - $\bullet$ Öffentlich-rechtliches Fernsehen

$\mathrm{N}=$ Fernsehberichte zu jährlich jeweils zwei Sexualmorden an Kindern. Eigene Grafik nach Schneider, Arnold und Greve (2005a).

Nach Albrecht weisen gerade Sexualmorde an Kindern alle Merkmale auf, „die zur Skandalisierung und entsprechender medialer Aufbereitung benötigt werden“ (Albrecht 2004: 506). Cohen zufolge löst sexuelle Gewalt an Kindern eine geradezu panikartige Wahrnehmung von Verletzlichkeit (panicky sense of vulnerability) aus (Cohen 2003: XVI).

\subsubsection{Vermittlung von Reichweite}

Kepplinger analysiert, dass statistische Maße als verlässlicher Ausdruck tatsächlicher Reichweite in der Kriminalitätsberichterstattung kaum eine Rolle spielen denn es wird vor allem über die schweren, aber seltenen Straftaten berichtet, während die leichten, aber häufigen Delikte in den Medien kaum aufgegriffen werden (Kepplinger 2000: 63, Davis 1952: 325-330, Baumann 1995: 25, Derwein 1995: 69ff.). Krüger ermittelt, dass in den Fernsehnachrichten der vier größten Sender 2007 Morde Thema von 42,4 Prozent aller Kriminalitätsberichte sind (Krüger 2008: 70). Demgegenüber machen Tötungsdelikte 0,01 Prozent der gesamten polizeilich bekannt gewordenen Kriminalität aus (BKA 2008: 268-291). ${ }^{8}$

8 Hier werden die Zahlen für alle vollendeten Tötungsdelikte - ohne fahrlässige Tötung - zum Vergleich herangezogen, da in der aktuellen Berichterstattung beispielsweise zwischen Mord, Totschlag und Körperverletzung mit Todesfolge in der Regel nicht unterschieden wird. 
Damit werden also Tötungsdelikte, die mit dem Blick auf die gesamte Kriminalität vergleichsweise wenige Menschen treffen, überportional oft aufgegriffen.

Wie wenig amtliche Statistiken die Entscheidungsbildung in den Redaktionen beeinflussen, weist Fishman (1980) nach, der eine Welle der Berichterstattung über ältere Verbrechensopfer in New York mitverfolgt. Ein Reporter schildert ihm, wie erstaunt er ist, dass die Polizei entgegen des medialen Trends abnehmende Fallzahlen regstriert. Für die redaktionelle Entscheidung erweist sich die Statistik als unbedeutend: "The whole issue was too big to pass up or play down" (Fishman 1980: 5). Dennoch nennen Journalistinnen und Journalisten bei einer OnlineBefragung den Nachrichtenfaktor Reichweite als wichtigstes Kriterium ihrer Nachrichtenauswahl, im Sinne einer tatsächlichen oder auch einer hypothetischen Betroffenheit (Ruhrmann und Göbbel 2007: 41). Zu fragen bleibt, ob in der Berichterstattung über Gewaltkriminalität Reichweite zwar bedeutsam ist, vor allem aber im Sinne von gefühlter Bedrohung. Möglicherweise also diktiert nicht die Statistik, sondern das Ausmaß der (vermuteten) Ängste des Publikums die journalistischen Auswahl- und Thematisierungsstrategien.

\subsubsection{Personalisierung}

Wilke (1984) weist mit seiner Analyse von Zeitungen aus vier Jahrhunderten nach, dass sich insbesondere Personalisierung im Längsschnitt dauerhaft nachweisen lässt - entgegen der Annahme von Galtung und Ruge (1965), wonach Personalisierung kulturabhängig ist. Neuere Studien konstatieren eine gewachsene Neigung zu personalisierter Berichterstattung, besonders im Fernsehen. Daschmann und Brosius (1997: 486) bezeichnen Fallbeispiele, mit denen Einzelschicksale und die Meinungen einzelner Personen beispielhaft dargestellt werden, als ,weit verbreitetes Stilmittel im deutschen Fernsehjournalismus“ (vgl. Daschmann 2001, 2007). Krüger und Zapf-Schramm (2003: 544f.) erkunden inhaltsanalytisch, dass personalisierte Fallbeispiele von Sendern unterschiedlich stark genutzt werden und von den Privatsendern RTL und Sat.1 besonders häufig präsentiert werden. Auch Maier, Ruhrmann und Klietsch (2006: 46) halten die Bedeutung von Personalisierung bei den Nachrichten der kommerziellen Sender für deutlich höher als bei den öffentlichrechtlichen Nachrichten.

Bolz sieht Personalisierung als eine wesentliche Darstellungsform, um Komplexität zu reduzieren, geradezu als inneren Zwang des Mediums - danach kann das Fernsehen gar nicht anders, als alles, worum es in der Welt geht, an Personen zu zeigen (Bolz 2006). Hickethier nennt Personalisierung ein wesentliches Element der Fernsehnachrichten, die er als Erzählungen von der Welt charakterisiert (Hickethier 2002, vgl. Lorenz 2002: 112-114). In boulevardesken Formen wird Personalisierung besonders stark wahrgenommen (Wegener 1994: 55, Mikos 1998: 68). 
Im Folgenden werden empirische Befunde zur Kriminalitätsberichterstattung daraufhin betrachtet, inwieweit sich die Konstruktion von Eindeutigkeit nachweisen lässt, im Sinne des von Galtung und Ruge (1965) beschriebenen Nachrichtenfaktors unambiguity und der von der weiteren Nachrichtenwertforschung meist implizit verwendeten Annahme, Schaden und Nutzen ließen sich klar von einander abgrenzen (Schulz 1976, Staab 1990, Kepplinger 1990, Maier, Ruhrmann und Klietsch 2006, Ruhrmann und Göbbel 2007). Im Kontext der theoretisch begründeten und empirisch belegten Personalisierung (s.o.) richtet sich der Blick vor allem darauf, wie über - in der Regel mutmaßliche - Täter und Täterinnen auf der einen Seite und Verbrechensoper auf der anderen berichtet wird. Die Polarisierung ist ein zentraler Aspekt medialer Vermittlung von Gewalt. „Crime stories deal with mythic issues of good and evil, personalized in the character of heroes and villains" (Cavender 2004: 338, vgl. Sacco 1995).

Mit der besonderen Aufmerksamkeit für Zerstörung (Galtung und Ruge 1965: 69f., Schulz 1976: 82, Kepplinger 1990: 41f., 2000: 72), korrespondiert die in früheren Studien vielfach analysierte Fokussierung auf zerstörerische Personen (Althoff 1999: 492). Scharf, Mühlenfeld und Stockmann stellen im Vergleich von fünf Zeitungen und jeweils zwei Wochenzeitungen und Nachrichtenmagazinen fest, dass in 35 Prozent der Beiträge über Kriminalität ausschließlich über Täter, Täterinnen bzw. Tatverdächtige berichtet wird, nicht aber über die Opfer. Lediglich drei Prozent der Beiträge widmen sich ausschließlich den Opfern (Scharf, Mühlenfeld und Stockmann 1999: 457, vgl. Hestermann 1997: 57, Hestermann 2009b, Baumann 1995: 11, Bielstein 1996: 1042, Obermöller und Gosch 1995).

Wie Baurmann darlegt, sind Verbrechensopfer in der US-amerikanischen Öffentlichkeit früher als in Deutschland in den Mittelpunkt des medialen Interesses gerückt (Baurmann 2004). So spricht Katz bereits 1987 vom Opfer als der zentralen Symbolfigur für die Herausforderung kollektiver Identität (Katz 1987: 52). Garland (2002: 11) sieht Verbrechensopfer als Schlüsselfiguren furchterregender Berichte über Gewalt. Friedrichsen (2004: 201) resümiert „die öffentliche Wiederentdeckung des Opfers“. Dabei gilt dieses Interesse keineswegs dem Opfer im Allgemeinen, sondern einem Idealbild eines Opfers. Das bevorzugte Opfer ist unschuldig und hilflos (Altheide 2002: 93) und besonders verletzlich (Katz 1987: 55). Dies führt zu der Frage, über welche Verbrechensopfer vorrangig berichtet wird.

\section{Kindliche Opfer erregen größtes Interesse}

Zenz beobachtet noch Ende der 70er Jahre, dass Gewalt an Kindern tabuisiert wird. Keine noch so grausame, selbst tödliche Kindesmisshandlung habe in der Bundesrepublik bis dahin mehr als Tagesaufmerksamkeit erregt (Zenz 1978: 21-23). Dieser Befund ist klar überholt. Proctor, Badzinksi und Johnson entwerfen eine Demografie der berichteten Kriminalität und sehen die klare Neigung, gehäuft über Gewalt an Kindern zu berichten (Proctor, Badzinksi und Johnson 2002: 360, vgl. Chermak 1995: 82). 
Derwein (1995: 137) analysiert die Berichterstattung der Frankfurter Allgemeinen Zeitung, der Süddeutschen Zeitung, der Bild-Zeitung und des Tagesanzeigers (einer in der Frankfurter Neuen Presse enthaltenen Lokalbeilage) im Jahre 1988. Dabei führt er die Altersstruktur der Opfer von Tötungsdelikten (Mord und Totschlag) auf und vergleicht sie mit den Zahlen der Polizeilichen Kriminalstatistik. Da Derwein seine statistischen Quellen nicht weiter spezifiziert, wurden seine Angaben nach den polizeilichen Statistiken des Jahres 1988 (BKA 1989: 97) neu berechnet. Daraus ergibt sich der klare Befund, dass über kindliche Opfer 2,8-mal so viel berichtet wird als es den statistischen Altersanteilen entspricht, dagegen über Menschen von 60 Jahren und älter nur halb so oft wie es ihrem Anteil an der Kriminalstatistik entspricht.

Tabelle 3: Alter der Opfer von Tötungsdelikten im Vergleich von Zeitungsberichterstattung und Polizeilicher Kriminalstatistik 1988

\begin{tabular}{|l|r|r|r|r|}
\hline Alter & \multicolumn{1}{|c|}{$\mathrm{n}$} & \multicolumn{1}{c|}{$\%$} & \multicolumn{1}{c|}{ PKS \% } & \multicolumn{1}{l|}{ Faktor } \\
\hline Unter 14 & 47 & 29,6 & 10,6 & 2,8 \\
\hline 14 bis 20 & 21 & 13,2 & 5,3 & 2,5 \\
\hline 21 bis 59 & 78 & 49,0 & 67,7 & 0,7 \\
\hline 60 und älter & 13 & 8,2 & 16,4 & 0,5 \\
\hline & 159 & 100 & 100 & \\
\hline
\end{tabular}

$\mathrm{N}=159$ Artikel über Mord und Totschlag, die Angaben zum Alter der Opfer enthielten, aus der Frankfurter Allgemeinen Zeitung, der Süddeutschen Zeitung, der Bild-Zeitung und des Tagesanzeigers (einer in der Frankfurter Neuen Presse enthaltenen Lokalbeilage), Untersuchungszeitraum 2. 5. bis 30.6. 1988 außer Süddeutsche Zeitung (1. 10.- 30.11. 1988). Eigene Berechnungen nach Derwein (1995: 137).

PKS $\%=$ Gewichteter Mittelwert der prozentualen Altersverteilung von Opfern bei vollendetem Mord, Totschlag und Tötung auf Verlangen im Jahre 1988. Eigene Berechnungen nach BKA (1989: 97).

Weibliche Opfer werden unterschiedlich stark beachtet

Proctor, Badzinksi und Johnson (2002: 360) legen dar, dass über die Gewalt an weiblichen Opfern verstärkt berichtet wird. Reijnders (2005) hält das junge, weibliche Opfer für eine Stereotype, derer sich moderne Fernsehformate ähnlich wie Balladen des 19. Jahrhunderts bedienen. Doch eine durchgehend überproportionale Beachtung weiblicher Opfer lässt sich empirisch nicht stützen. Nach einer Analyse 
von Fernsehnachrichten sind Gewaltopfer in 1,9 Prozent der Berichte überwiegend oder ausschließlich weiblich, in 72,4 Prozent sind sie überwiegend oder ausschließlich männlich (Groebel und Gleich 1993: 104). In ihrer elf Jahre später durchgeführten Analyse von Fernsehgewalt im weitesten Sinne ist der Anteil weiblicher Opfer mit 27,5 Prozent deutlich höher (Grimm, Kirste und Weiß 2005: 198).

Eine Inhaltsanalyse der Chicago Tribune ergibt einen Wert von 37,3 Prozent weiblicher Kriminalitätsopfer (Graber 1980: 56). Eine Analyse deutscher Tageszeitungen kommt zu einem ähnlichen Wert: Berichten die Frankfurter Allgemeinen Zeitung, die Süddeutsche Zeitung, die Bild-Zeitung und der Tagesanzeiger im Jahr 1988 über Mord und Totschlag, werden bei 34,2 Prozent der Berichte weibliche Opfer genannt - und damit weniger als die Polizeiliche Kriminalstatistik mit 52,8 Prozent angibt (Derwein 1995: 130, BKA 1989: 97). Damit verweisen Inhaltsanalysen darauf, dass über weibliche Opfer keineswegs häufiger als über männliche Opfer berichtet wird. Auffällig sind die deutlichen Schwankungen in der Geschlechterverteilung.

Stigmatisierung des Täters bzw. der Täterin bzw. des/r Tatverdächtigen

Die Befunde zur Wiederentdeckung des Opfers in der Gewaltberichterstattung werfen die Frage auf, inwieweit ihr eine Stigmatisierung derer gegenübersteht, die für Gewalt verantwortlich gemacht werden. Dies wird widersprüchlich diskutiert. So wird unterstellt, dass die Medien das Ziel verfolgen, den gesellschaftlichen Wertekonsens zu festigen, indem sie die Empörung des Publikums über Straftäter und Straftäterinnen erregen (Brüchert 2005: 21). Bolz hält Stigmatisierung für ein wichtiges Stilmittel des Fernsehens: „Man zeigt die Bösen, die Verantwortlichen, die Skandalnudeln, und auf die kann man dann seine Verachtung konzentrieren“ (Bolz 2006, vgl. Burkhart 2002, Obermöller und Gosch 1995: 45-47). Kaiser (2002: 31) dagegen sieht keine Anzeichen exzessiver Stigmatisierung.

Tatsächlich hält die pauschale Unterstellung, die Medien neigten dazu, Tatverdächtige bzw. Verurteilte zu stigmatisieren, keiner empirischen Überprüfung stand. Derwein (1995: 125f.) geht der Frage nach, inwieweit in Kriminalitätsberichten der Frankfurter Allgemeinen Zeitung, der Süddeutschen Zeitung, der Bild-Zeitung und des Tagesanzeigers (einer in der Frankfurter Neuen Presse enthaltenen Lokalbeilage) Tatverdächtige herabgewürdigt werden. Dafür findet er nur bei 3,1 Prozent von insgesamt 1544 Artikeln einen Beleg. Eine offene Stigmatisierung spielt nach Erkenntnis von Grimme, Kirste, und Weiß (2005: 214) auch in dokumentarischen Formaten des Fernsehens praktisch keine Rolle.

Abweichende Ergebnisse erbringt die Analyse von boulevardesken Medienformaten. Mikos (1998: 69) stellt bei TV-Boulevardmagazinen fest, dass mit der Übernahme der Opferperspektive und der Beschreibung der Tatfolgen für Opfer und Angehörige Anklage gegen die Täter erhoben wird. „Dabei wurde in den BoulevardMagazinen großer Wert auf die moralische Verurteilung der Täter gelegt.“ Auch Grimm, Kirste und Weiß (2005: 243) beobachten in Boulevardmagazinen „vielfach Inszenierungsstrategien, die das Urteil der Zuschauer lenken und zumeist auf eine emotional begründete Verurteilung der Täter abzielen“. Schneider, Arnold und 
Greve (2005a) ermitteln, dass in der Berichterstattung über Sexualmorde an Kindern die Boulevardpresse als einziges der untersuchten Mediengenres in rund jedem dritten Beitrag (32,2 Prozent) dazu neigt, Tatverdächtigen eine Schuld zuzuschreiben. Im Privatfernsehen geschieht dies kaum (2,4 Prozent) und im öffentlich-rechtlichen Fernsehen in keinem der untersuchten Fälle.

\section{Stigmatisierung ausländischer Tatverdächtiger}

Eine Reihe von Untersuchungen widmet sich der Frage, ob ausländische Tatverdächtige in besonderer Weise stigmatisiert werden. Dies wird überwiegend bejaht. Galanis (1987) wirft Printmedien eine einseitige Berichterstattung über Eingewanderte vor. Bohn, Hamburger und Rock kritisieren eine Lokalberichterstattung, die Sinti und Roma fast nur dann in den Blick nimmt, wenn sie unter Tatverdacht stehen (Bohn, Hamburger und Rock 1995: 166-183, vgl. Jäger, Ruth, Jäger und Cleve 1998). Eine verzerrte Darstellung von Minderheiten stellen Dixon und Linz fest. Ihnen zufolge tauchen Schwarze und Lateinamerikaner in Fernsehnachrichten US-amerikanischer Sender vor allem als Tatverdächtige, seltener als Opfer auf (Dixon und Linz (2000: 547-573, vgl. Welch 2007: 276, Oliver 1994).

Einige Studien kommen zu gegenteiligen Befunden. Carter ermittelt in einer experimentellen Studie, dass Studierende, die einen Bericht über einen gewalttätigen Einbruch schreiben, in geringerem Maße von der Schuld eines Tatverdächtigen ausgehen, wenn sie ihn für dunkelhäutig halten, als wenn sie davon ausgehen, dass er weiß ist (Carter 1959). Nach Derwein wird in drei der vier untersuchten Tageszeitungen im Vergleich zur Polizeistatistik eher unterproportional oft über ausländische Tatverdächtige berichtet, lediglich in der Bild-Zeitung leicht überproportional (Derwein 1995: 112). Saleth zieht bei ihrer Untersuchung der Lokalberichterstattung des Schwäbischen Tagblatts zwischen 1975 und 2000 das Fazit: „Eine deutlich negativ gefärbte Darstellung ausländischer Tatverdächtiger konnte nicht festgestellt werden“" (Saleth 2004: 145).

\subsubsection{Visualisierung}

Schulz beschreibt eine zunehmende Bildlastigkeit der Medien, die eine „Konzentration auf sensationelle und dramatische Ereignisse" fördere (Schulz 1996: 7, vgl. Hacker 1988: 21). Bielstein (1996: 1042) sieht einen Trend ,zur Visualisierung, zum spannend gemachten ,Reality-TV'“. In den 60er Jahren kommt Buckalew noch zu anderen Ergebnissen, als er 12 Fernsehschaffende 64 verschiedene Nachrichtenthemen bewerten lässt $-\mathrm{zu}$ einigen gibt es Bildmaterial, zu anderen keines. Jene Themen, zu denen Bilder verfügbar sind, werden nur leicht bevorzugt (Buckalew 1969, vgl. Staab 1998: 55). Dagegen stellt die Forschungsgruppe um Ruhrmann in der Längsschnittanalyse deutscher Fernsehnachrichten zwischen 1992 und 2004 eine wachsende und themenübergreifende Bedeutung von Visualität fest (Maier, Ruhrmann und Klietsch 2006: 11, vgl. Diehlmann 2003: 144). Dies zeigt sich im fast 
völligen Verschwinden nur gesprochener und nicht illustrierter Meldungen. Für 70 Prozent der öffentlich-rechtlichen Nachrichten und 97 Prozent der Privat-TV-Nachrichten im Jahr 2004 wird Visualität als bedeutsam codiert (Maier, Ruhrmann und Klietsch 2006: 48).

Damit ist die Frage aufgeworfen, welche spezifischen Folgen diese wachsende Bedeutung von Visualität für die Fernsehberichterstattung über Gewaltkriminalität hat. Einige Studien begründen kategorisch einen fernsehtypischen Hunger besonders nach Gewaltbildern. Plake zeigt sich überzeugt: „Ideal für das Medium Fernsehen ist ein Geschehen, das, wie Kriege, Überfälle, terroristische Anschläge usw., mit spannungsgeladener Aktion einher geht.“ (Plake 2004: 112) Damit einer geht die Annahme, aggressive Handlungen seien sehr einfach zu visualisieren (Bruns 1998: 12). Begründet wird diese These mit dem intimen, expressiven Format des Fernsehens (Cavender 2004: 336f., vgl. Kerbel 2000: 14, Winterhoff-Spurk 1986: 130). Groebel und Gleich (1993: 20) stellen kategorisch fest: Gewalt „macht visuell mehr her, besteht aus kamera-freundlichen schnellen Bewegungen und bietet eben einfach höheren Nervenkitzel“".

Eine Reihe von Studien belegt empirisch, dass mit einer gewachsenen Visualisierung in der Fernsehberichterstattung Gewaltbilder an Bedeutung gewonnen haben (Maier, Ruhrmann und Klietsch 2006: 12, Bruns und Marcinkowski 1997: 227f.). Winterhoff-Spurk, Unz und Schwab berechnen im Vergleich der Hauptnachrichtensendungen von ARD, ZDF, RTL, SAT.1 und ProSieben eine Steigerung des Anteils von 21 Prozent gewalthaltiger Beiträge im Jahr 1996 auf 31 Prozent im Jahr 2002, nach einem Zwischenhoch von 34 Prozent in den Jahren 1998 und 2000 (Winterhoff-Spurk, Unz und Schwab 2005: 225-237).

Die These allerdings, dass eine zunehmend visualisierte Berichterstattung bedeutet, Gewalttaten selbst oder die unmittelbaren Folgen zu zeigen, beispielsweise Tod und Verletzung (Plake 2004, Groebel und Gleich 1993), lässt sich für die aktuelle Berichterstattung über Gewaltkriminalität nicht stützen. So sind in dokumentarischen Formaten - anders als in fiktionalen Sendungen - Bilder der Gewalttat selbst kaum verfügbar (Mikos 2003: 12). Bettina Warken, Chefin der ZDF-Nachrichten, schildert ihren Eindruck, dass drastische Gewaltbilder zumindest in der eigenen Redaktion immer strenger herausgefiltert werden (Warken 2006). So lässt sich empirisch zwar belegen, dass Gewaltkriminalität zunehmend bebildert wird, dabei wird aber die Tat in der Regel nur mittelbar visualisiert. Gezeigt werden vermehrt der Tatort, die Arbeit der Spurensicherung danach oder auch Porträts der Opfer (Bruns und Marcinkowski 1997: 227f.). Zum gleichen Ergebnis kommen Grimm, Kirste und Weiß. Nach ihrer Analyse wird in der Regel das Geschehen nach der Gewalttat gezeigt, also beispielsweise, wie unmittelbar bzw. mittelbar Beteiligte die Tat und ihre Auswirkungen erleben (Grimm, Kirste und Weiß 2005: 88). 
Abb. 6: Visualisierung von Tätern und Täterinnen bzw. Tatverdächtigen und Opfern in Nachrichten und politischen Magazinsendungen

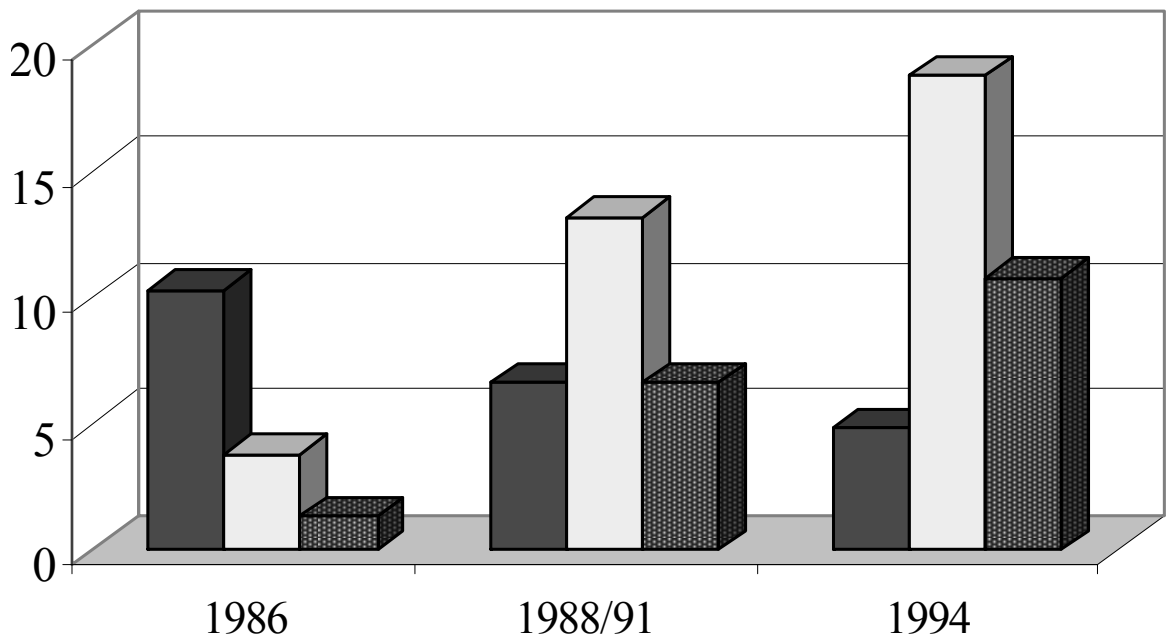

$\square$ Täter $\quad \square$ Opfer $\quad$ Täter und Opfer

Prozentualer Anteil der Filmberichte (ohne Sprechermeldungen), die Täter und Täterinnen bzw. Tatverdächtige und / oder Opfer in Nachrichten und politischen Magazinen ausgewählter Fernsehsender visualisieren. Datenbasis: ARD, ZDF, RTL und Sat.1 (jeweils eine Programmwoche in 1986 und 1988), ARD, ZDF, RTL, Sat.1 und ProSieben (eine Programmwoche in 1991), ARD, ZDF, RTL, Sat.1, ProSieben, n-tv, Vox und RTL2 (vier Programmwochen in 1994). Die Zahlen für 1988 und 1991 sind im Original zusammengefasst. Eigene Grafik nach Bruns (1998: 178).

Die zunehmende Beachtung von Opfern im Fernsehen zeigt sich auch in ihrer Visualisierung, ermittelt Bruns im Längsschnittvergleich von Fernsehnachrichten und politischen Magazinen öffentlich-rechtlicher und privater Fernsehsender aus sieben Programmwochen der Jahre 1986, 1988, 1991 und 1994. 1986 ist der Anteil der Filme, die ausschließlich die Tatverdächtigenseite zeigen, dreimal so hoch (10,3 Prozent) wie der Anteil der Filme, in denen nur das Opfer zu sehen ist (3,8 \%). 1994 hat sich das Verhältnis umgekehrt: Viermal so oft ist allein das Opfer zu sehen $(18,8$ $\%$ ) wie der oder die Tatverdächtige (4,8 \%). Zugleich steigt der Anteil der Filme mit beiden Seiten von 1,3 auf 10,8 Prozent (Bruns 1998: 177f.). Auch wenn die Analyse der Daten dadurch erschwert ist, dass die Angaben zu den ersten drei genannten Jahren jeweils auf lediglich einer Programmwoche basieren und die Auswahl der untersuchten Fernsehsender zweimal erweitert wurde, zeigt sich darin jedoch der Trend einer Verschiebung der Gewichte. 
Kriminalitätsberichterstattung in Massenmedien ist traditionsreich - frühe Abhandlungen darüber gehen bis ins 17. Jahrhundert, systematische Inhaltsanalysen ins 19. Jahrhundert zurück. Der Anteil des Themenfeldes schwankt erheblich, etwa an USamerikanischen nationalen Fernsehnachrichten zwischen 16,4 Prozent im Jahre 1995 und 1,8 Prozent im Jahre 2008. Durch Veränderungen der polizeilich registrierten Kriminalität lassen sich diese Schwankungen kaum erklären. Damit stellt sich die Frage, welche anderen Erklärungen herangezogen werden können.

In der Bundesrepublik gewichten die Nachrichten öffentlich-rechtlicher und privater Fernsehsender Gewaltkriminalität unterschiedlich - die Hauptnachrichten von Sat.1 und RTL berichten anteilig dreimal so umfangreich wie die Nachrichten von ARD und ZDF. Bei den Boulevardmagazinen dagegen thematisieren die öffentlich-rechtlichen Sender Gewaltkriminalität stärker als ihre private Konkurrenz. Zu erkunden ist, mit welchen Überlegungen diese Unterschiede zu erklären sind.

Als bedeutsames Kriterium der Berichterstattung erweist sich Folgenschwere im Sinne körperlicher Verletzung - drastische Gewalt wird überproportional berichtet. Etwa jeder zweite Mensch, über den das Fernsehen als Opfer krimineller Gewalt berichtet, ist zu Tode gekommen. Sexuelle Gewalt wird besonders beachtet. Reichweite im Sinne einer statistischen Verbreitung von Verbrechen spielt praktisch keine Rolle, aufgegriffen werden vor allem seltene Delikte. Offen bleibt, wie bedeutsam Reichweite als subjektive Größe ist.

Personalisierung lässt sich im Längsschnitt dauerhaft nachweisen und ist im Fernsehjournalismus ausgeprägt. In der Kriminalitätsberichterstattung ist sie als Verdichtung gewalttätiger Konflikte auf einzelne Leitfiguren zu beobachten. Mit ihr eng verknüpft ist die Vermittlung von Eindeutigkeit, insbesondere in der Darstellung von Verbrechensopfern auf der einen Seite und Tatverdächtigen bzw. Täterinnen und Tätern auf der anderen Seite. Deuten frühere Untersuchungen auf eine täterorientierte Berichterstattung hin, steht neueren Studien zufolge das Opfer im Mittelpunkt des medialen Interesses. Hierbei wird vor allem die Gewalt an Kindern fokussiert. Eine überproportionale Beachtung weiblicher Opfer ist sich nicht nachgewiesen. Vorwürfe an die Medien, sie neigten zu einer Stigmatisierung von Tatverdächtigen bzw. Verurteilten, lassen sich für das deutsche Fernsehen nicht generell bestätigen. Uneinheitlich wird die Frage beantwortet, ob Tatverdächtige ausländischer Herkunft bzw. dunkler Hautfarbe in besonderer Weise stigmatisiert werden. Während vor allem Studien aus den USA ergeben, dass Angehörige von Minderheiten eher als Tatverdächtige und seltener als Opfer dargestellt werden, zeigen andere Untersuchungen, dass Minderheiten nicht diskriminiert oder in der Berichterstattung gar bevorzugt werden. $\mathrm{Zu}$ erkunden ist, welche Überlegungen Journalistinnen und Journalisten bei der Auswahl der dargestellten Personen zugrunde legen und inwieweit diese Überlegungen handlungsrelevant sind.

Für das Fernsehen ist eine wachsende Bedeutung von Visualität belegt - kaum noch eine Meldung wird in den Nachrichten verlesen, ohne dass es bewegte Bilder dazu gibt. Inhaltsanalysen deuten auf eine gewachsene Illustration von Gewalt- 
themen. Belegen Inhaltsanalysen der 1980er Jahre, dass dies in Informationssendungen der bundesdeutschen Privatsender besonders ausgeprägt war, sind die Unterschiede zu öffentlich-rechtlichen Sendungen in jüngerer Zeit geringer geworden. In einer Reihe von Studien wird behauptet, Gewalt sei von hoher visueller Attraktivität. In der Kriminalitätsberichterstattung ist aber vor allem eine mittelbare Visualisierung festzustellen: In der Regel wird das Geschehen nach der Gewalttat gezeigt. Damit bleibt die Frage offen, welche Bilder Fernsehschaffenden als besonders publikumsaffin gelten, um Gewalt darzustellen, und welche Muster der Berichterstattung sich daraus ableiten lassen.

\subsection{Dimensionen des Erkenntnisinteresses}

Die Synopse des bisherigen Forschungsstandes liefert erste Befunde zur übergeordneten Frage: Welches Publikumsbild leitet Journalistinnen und Journalisten, wenn sie im Fernsehen über Gewaltkriminalität berichten? Diese Leitfrage soll systematisch empirisch beantwortet werden, indem sich diese Untersuchung auf folgende fünf Dimensionen konzentriert:

1. Forschungsfrage: Wie stark sind Fernsehjournalistinnen und Fernsehjournalisten an ihrem Publikum orientiert?

Wurde in der frühen Forschung der Einfluss des Publikums auf journalistische Entscheidungsprozesse als gering betrachtet, wird für die deutschen Medien seit Anfang der 1990er Jahre eine zunehmende Bedeutung des Publikums festgestellt. Im Kontext der vorliegenden Untersuchung soll die Intensität dieser Publikumsorientierung erforscht werden. Mit einer zunehmenden Publikumsorientierung konstatiert die Medienforschung einen gesunkenen Stellenwert journalistischer Handlungsabsichten, die nicht aus Publikumsbedürfnissen, sondern aus subjektiven oder geradezu missionarischen Haltungen der Medienschaffenden zu erklären sind. Zur Klärung der Relevanz von Publikumsorientierung wird daher auch erkundet: Wie stark spielen Handlungsabsichten eine Rolle, die nicht auf eine Publikumsorientierung deuten? Aus dieser ersten Dimension des Forschungsgegenstandes folgen Programmfragen für die Interviews mit Journalistinnen und Journalisten (s. Tab. 4). Der verwendete Gesprächsleitfaden und das Codebuch der Inhaltsanalyse sind im Anhang dokumentiert. 
Tabelle 4: Dimension des Erkenntnisinteresses: Publikumsorientierung

\begin{tabular}{|l|l|}
\hline Forschungsfrage & $\begin{array}{l}\text { Wie stark sind Fernsehjournalistinnen und Fernsehjour- } \\
\text { nalisten an ihrem Publikum orientiert? }\end{array}$ \\
\hline Interviewfragen & Stellenwert des Publikums: 3.1 \\
\cline { 2 - 2 } & $\begin{array}{l}\text { Absichten, unabhängig von Publikumsorientierung } \\
\text { generell etwas zu bewirken: } 6.1\end{array}$ \\
\cline { 2 - 2 } & $\begin{array}{l}\text { Absichten, unabhängig von Publikumsorientierung je- } \\
\text { weils auf die Ermittlungsbehörden, die Justiz, Verurteilte, } \\
\text { die Bevölkerung oder die Politik einzuwirken: 6.2-6.5 }\end{array}$ \\
\hline
\end{tabular}

2. Forschungsfrage: Wie machen sich Fernsehjournalistinnen und Fernsehjournalisten ein Bild von ihrem Publikum?

Der dynamisch-transaktionale Ansatz von Früh und Schönbach (1982, 1984, 2005), der journalistisches Handeln aus dem Wechselspiel mit dem Publikum erklärt, soll einer empirischen Überprüfung unterzogen werden. Dabei gilt es, das Konstrukt eines Para-Feedbacks zu erhellen, das Früh und Schönbach als zentralen Wirkungsfaktor beschreiben und das sich ihrem Konzept zufolge weniger aus tatsächlichen Reaktionen der Zuschauenden, sondern vor allem aus journalistischen Annahmen über das Publikum speist. Zugleich stellt sich die Frage, welche Rolle die Orientierung an anderen Kolleginnen und Kollegen im journalistischen Entscheidungsprozess spielt und inwiefern sie dazu dient, sich Klarheit über Publikumsbedürfnisse zu verschaffen, oder ob sie einer Publikumsorientierung zuwiderläuft. Bei der Analyse, wie ein journalistisches Publikumsbild entsteht, soll auch die Bedeutung der Messwerte der Sehbeteiligung und der Medienforschung in Betracht gezogen werden. Aus dieser zweiten Dimension des Forschungsgegenstandes sind die in Tab. 5 aufgeführten Programmfragen für die Interviews mit Journalistinnen und Journalisten entwickelt worden.

Tabelle 5: Dimension des Erkenntnisinteresses: Publikumsbild

\begin{tabular}{|l|l|}
\hline Forschungsfrage & $\begin{array}{l}\text { Wie machen sich Fernsehjournalistinnen und } \\
\text { Fernsehjournalisten ein Bild von ihrem Publikum? }\end{array}$ \\
\hline Interviewfragen & $\begin{array}{l}\text { Resonanz des Publikums, Bedeutung und Messung der } \\
\text { Sehbeteiligung: 3.2 }\end{array}$ \\
\cline { 2 - 2 } & $\begin{array}{l}\text { Vermutete Erfolgsfaktoren und Misserfolgsfaktoren: } 3.3 \\
-3.4\end{array}$ \\
\hline
\end{tabular}


3. Forschungsfrage: Von welchen Publikumsbedürfnissen hinsichtlich der Berichterstattung über Gewaltkriminalität gehen Fernsehschaffende aus?

Zunächst soll die vielfach erhobene These, Gewaltberichterstattung sei per se ein Publikumsmagnet, überprüft werden. Daran schließen sich spezifizierende Fragen danach an, welche Merkmale der Berichterstattung aus journalistischer Sicht in besonderem Maße das Interesse des Publikums wecken und welche Wirkungen dabei vermutet werden. Auch diese dritte Dimension des Forschungsgegenstandes führt zu Programmfragen, die ausschließlich durch Interviews mit Fernsehjournalistinnen und Fernsehjournalisten beantwortet werden sollen (s. Tab. 6).

Tabelle 6: Dimension des Erkenntnisinteresses: vermutete Publikumsbedürfnisse

\begin{tabular}{|l|l|}
\hline Forschungsfrage & $\begin{array}{l}\text { Von welchen Publikumsbedürfnissen hinsichtlich der } \\
\text { Berichterstattung über Gewaltkriminalität gehen } \\
\text { Fernsehschaffende aus? }\end{array}$ \\
\hline Interviewfragen & $\begin{array}{l}\text { Bedeutung des Gebrauchswertes der Berichterstattung: } \\
2.6\end{array}$ \\
\cline { 2 - 3 } & $\begin{array}{l}\text { Konkrete Erfahrungen mit Publikumsresonanz und } \\
\text { Folgerungen daraus: } 3.2-3.4\end{array}$ \\
\cline { 2 - 3 } & $\begin{array}{l}\text { Bedeutung von Gewalt in der Berichterstattung: 3.6 - } \\
3.7\end{array}$ \\
\cline { 2 - 3 } & $\begin{array}{l}\text { Annahmen zur Wirkung emotionalisierter Bericht- } \\
\text { erstattung: } 4.6\end{array}$ \\
\cline { 2 - 3 } & Annahmen zur Wirkung von Personalisierung: 3.5 \\
\hline
\end{tabular}

4. Forschungsfrage: Welche Auswahl- und Thematisierungskriterien folgern Fernsehschaffende aus ihren Annahmen über die Bedürfnisse ihres Publikums?

Zunächst stellt sich die Frage, welche Kriterien aus journalistischer Sicht bei der Auswahl und Thematisierung im Vordergrund stehen. Aus der konstruktivistischen Betrachtungsweise dieser Untersuchung heraus geht es damit nicht um objektive Ereignismerkmale, sondern um die journalistische Wahrnehmung und Bewertung von Ereignissen mit Blick auf die angenommenen Maßstäbe des Publikums. Zu erfragen ist, welches Gewicht die Emotionalisierung des Publikums aus journalistischer Sicht hat - beispielsweise in der Abwägung statistisch messbarer Ereignismerkmale gegenüber gefühlten Ereignismerkmalen. Daran schließen sich Fragen zu Mustern der Thematisierung an. 
Tabelle 7: Dimension des Erkenntnisinteresses: Auswahl- und Thematisierungskriterien

\begin{tabular}{|l|l|}
\hline Forschungsfrage & $\begin{array}{l}\text { Welche Auswahl- und Thematisierungskriterien folgern } \\
\text { Fernsehschaffende aus ihren Annahmen über die Be- } \\
\text { dürfnisse ihres Publikums? }\end{array}$ \\
\hline Interviewfragen & Leitkriterien allgemein: $2.3-2.4$ \\
\cline { 2 - 3 } & Emotionalisierung: $4.1-4.5$ \\
\cline { 2 - 3 } & Vermittlung von Folgenschwere: 4.8 \\
\cline { 2 - 3 } & Vermittlung von Reichweite: $2.5,2.6$ \\
\cline { 2 - 3 } & Personalisierung: 3.5 \\
\cline { 2 - 3 } & Vermittlung von Eindeutigkeit: $3.5,3.7,4.6-4.8$ \\
\hline & Visualisierung: $5.1-5.2$ \\
\hline
\end{tabular}

5. Forschungsfrage: Inwieweit sind die beschriebenen journalistischen Auswahlund Thematisierungskriterien handlungsrelevant?

Als fünfte Dimension soll das tatsächliche Handeln von Fernsehjournalistinnen und Fernsehjournalisten in ihrer Berichterstattung über Gewaltkriminalität erkundet werden. Dabei geht es sowohl darum, wie Journalistinnen und Journalisten ihre Handlungsmuster selbst beschreiben, als auch darum, welche dieser Muster sich in der Inhaltsanalyse von Beiträgen über Gewaltkriminalität in Nachrichten und Boulevardmagazinen der reichweitenstärksten deutschen Fernsehsender abbilden.

Zunächst ist zu fragen, welchen Stellenwert das Thema Gewaltkriminalität insgesamt einnimmt, indem der Umfang die Kriminalitätsberichterstattung im jeweiligen redaktionellen Arbeitsalltag der Befragten und in den untersuchten Sendungen erkundet wird. Zu erforschen ist weiter, inwieweit sich in den Selbstbeschreibungen und der Inhaltsanalyse Strategien der Emotionalisierung erkennen lassen. Schließlich stehen die nach bisheriger Erkenntnis zentralen Nachrichtenfaktoren im Fokus: Welche Rolle spielt die Folgenschwere insbesondere im Sinne gravierender bzw. tödlicher Verletzung sowie sexueller Gewalt? Wie bedeutsam ist die Reichweite im Sinne einer statistischen Verbreitung von Verbrechen oder der gefühlten Betroffenheit? Wie stark lässt sich eine Personalisierung nachweisen, über welche Personen wird wie berichtet? Wie stark ist Eindeutigkeit als Nachrichtenfaktor, insbesondere was die Darstellung von Verbrechensopfern vs. Tatverdächtigen bzw. Verurteilten 
angeht, und welche Personenmerkmale sind dabei jeweils besonders relevant? Welche Rolle spielt schließlich die Visualisierung, wie werden das Tatgeschehen und die handelnden Personen gezeigt? Die Operationalisierung aller Forschungsfragen wird in Kapitel 4 beschrieben. Die Antworten auf die Forschungsfragen sind in Kapitel 5 aufgeführt.

Tabelle 8: Dimension des Erkenntnisinteresses: journalistisches Handeln

\begin{tabular}{|c|c|}
\hline Forschungsfrage & $\begin{array}{l}\text { Inwieweit sind die beschriebenen journalistischen } \\
\text { Auswahl- und Thematisierungskriterien handlungs- } \\
\text { relevant? }\end{array}$ \\
\hline \multirow[t]{2}{*}{ Interviewfragen } & $\begin{array}{l}\text { Stellenwert des Themas Gewaltkriminalität im } \\
\text { Berufsalltag: } 1.1-1.2\end{array}$ \\
\hline & $\begin{array}{l}\text { Beschreibung und Reflexion journalistischen Handelns: } \\
2.1-2.2 \text { sowie jeweils } \mathrm{zu} \text { den aufgezeichneten } \\
\text { Beiträgen der Befragten }\end{array}$ \\
\hline \multirow{7}{*}{$\begin{array}{l}\text { Kategorien der } \\
\text { Inhaltsanalyse }\end{array}$} & Umfang der Berichterstattung: $1.1-1.4$ \\
\hline & $\begin{array}{l}\text { Emotionalisierung: } 1.7-1.13,2.2-2.24,3.2-3.25 \text {, } \\
4.7,4.9-4.10,4.12,4.13,4.15,4.16,4.18,4.19,4.21 \text {, } \\
4.22,4.24,4.25,4.27,4.28,4.30,5.1-5.4\end{array}$ \\
\hline & $\begin{array}{l}\text { Vermittlung von Folgenschwere: } 1.1-1.4 ., 1.7 .-1.13 \text {, } \\
2.1,2.3-2.8,2.10-2.24,3.1,3.15,3.16,3.19,3.22- \\
3.24,4.1-4.6\end{array}$ \\
\hline & $\begin{array}{l}\text { Vermittlung von Reichweite: } 1.1-1.4,1.13,2.3-2.8 \text {, } \\
2.23,2.24,3.1,3.3-3.8,3.10,3.23,3.24,4.1-4.6,5.1 \\
-5.4\end{array}$ \\
\hline & $\begin{array}{l}\text { Personalisierung: } 1.10-1.12,2.1-2.24,3.1-3.25,4.1 \\
-4.6,4.10-4.12,4.16-4.18,4.22-4.24,4.28-4.30\end{array}$ \\
\hline & $\begin{array}{l}\text { Vermittlung von Eindeutigkeit: } 1.1-1.4,1.7-1.12,2.2 \\
-2.24,3.2-3.25,4.1-4.30\end{array}$ \\
\hline & $\begin{array}{l}\text { Visualisierung: } 1.10-1.12,2.11-2.14,2.18-2.20 \text {, } \\
\text { 2.22., } 2.24,3.11-3.14,3.16,3.20-3.22,3.24,4.1- \\
4.6,4.10-4.12,4.22-4.24,4.28-4.30\end{array}$ \\
\hline
\end{tabular}

